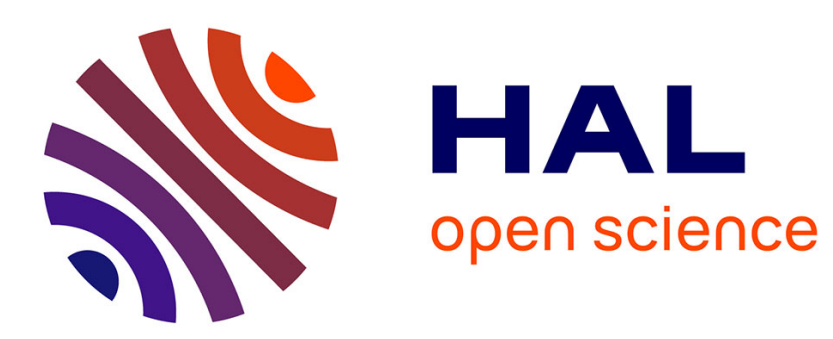

\title{
On modelling brick/mortar interface via a St. Venant-Kirchhoff orthotropic soft interface. Part I: theory
}

\author{
Maria Letizia Raffa, Frédéric Lebon, Raffaella Rizzoni
}

\section{To cite this version:}

Maria Letizia Raffa, Frédéric Lebon, Raffaella Rizzoni. On modelling brick/mortar interface via a St. Venant-Kirchhoff orthotropic soft interface. Part I: theory. International Journal of Masonry Research and Innovation, 2016, 1, pp.142 - 142. 10.1504/IJMRI.2016.077470 . hal-01478964

\author{
HAL Id: hal-01478964 \\ https://hal.science/hal-01478964
}

Submitted on 28 Feb 2017

HAL is a multi-disciplinary open access archive for the deposit and dissemination of scientific research documents, whether they are published or not. The documents may come from teaching and research institutions in France or abroad, or from public or private research centers.
L'archive ouverte pluridisciplinaire HAL, est destinée au dépôt et à la diffusion de documents scientifiques de niveau recherche, publiés ou non, émanant des établissements d'enseignement et de recherche français ou étrangers, des laboratoires publics ou privés. 


\title{
On modelling brick/mortar interface via a St. Venant-Kirchhoff orthotropic soft interface. Part I: theory
}

Maria Letizia Raffa*

LMA-CNRS UPR 7051,

Aix-Marseille University,

4 Impasse Nikola Tesla CS 40006 - 13453,

Marseille Cedex 13, France

Email: raffa@lma.cnrs-mrs.fr

*Corresponding author

\section{Frédéric Lebon}

LMA-CNRS UPR 7051,

Aix-Marseille University,

Centrale Marseille,

4 Impasse Nikola Tesla CS 40006 - 13453,

Marseille Cedex 13, France

Email: lebon@lma.cnrs-mrs.fr

\section{Raffaella Rizzoni}

Department of Engineering, University of Ferrara, via Saragat 1 - 44121 Ferrara, Italy

Email: raffaella.rizzoni@unife.it

\begin{abstract}
In this paper, a nonlinear-imperfect interface model is proposed in order to model brick/mortar-interface behavior in small masonry assemblies. The proposed model, formulated according a micromechanical strategy, derives from a consolidated approach coupling arguments of asymptotic analysis and homogenisation method. The adopted asymptotic technique is extended to the finite strain theory. The homogenisation strategy, under the non-interacting approximation, is extended to microcracked-orthotropic-hyperelastic materials. Simple numerical simulations, developed within the framework of finite element method, highlight the model soundness and its applicability in finite-strain problems.
\end{abstract}

Keywords: asymptotic analysis; finite elasticity; imperfect interface; masonry structures; orthotropic interphase; thin film.

Reference to this paper should be made as follows: Raffa, M.L., Lebon, F and Rizzoni, R. (2016) 'On modelling brick/mortar interface via a St. VenantKirchhoff orthotropic soft interface. Part I: theory', Int. J. Masonry Research and Innovation, Vol. 1, No. 2, pp.142-164. 
Biographical notes: Maria Letizia Raffa, $\mathrm{PhD}$, is a Teaching and Research Temporary Attaché at the Aix-Marseille University, France. She received her Master's degree in Civil Engineering from University of Messina, Italy. She received her Doctorate in Joint Supervision in Civil Engineering from University of Rome 'Tor Vergata' (Italy) and in Solid Mechanics from Aix-Marseille University (France), with a thesis entitled "Micromechanical modeling of imperfect interfaces and applications". Her current research interests include micromechanical homogenization techniques for heterogeneous media, interface modelling with applications in civil engineering, biomechanics and contact mechanics.

Frédéric Lebon, $\mathrm{PhD}$, Hab is a full-time professor of Solids Mechanics at AixMarseille University, France. He received his Master's degree and his $\mathrm{PhD}$ in Applied Mathematics in Marseille University and the Habilitation in Mechanics in Montpellier University. He is actually head of the Mechanics and Acoustics Laboratory in Marseille. His work focuses on computational mechanics, contact mechanics, interface modelling and non-smooth mechanics. He published more than 70 papers in international journals and more than 100 proceedings of conferences. His h-factor is 14 (Scopus)/18 (Scholar).

Raffaella Rizzoni, PhD, is an Assistant Professor of Solid Mechanics at the University of Ferrara, Italy. She received the Engineering of Materials degree at the University of Ferrara and her $\mathrm{PhD}$ in Structural Engineering at the University of Florence. Her work focuses on continuum mechanics, elasticity, thin structures and smart materials. Her research activity is documented by more than 50 papers, published in journals and proceedings of national and international conferences. Her actual h-index is 8 (Scopus source, February 2016).

\section{Introduction}

Interfaces play a fundamental role in the mechanical strength of a masonry structure. They represent a zone of weakness in which the localisation of high stresses may activate microcracks nucleation and they are the principal planes of microcracks propagation in many loading cases. Generally, two classical approaches in interfaces modelling can be distinguished. The first one, called phenomenological method, is based on experimental evidences and the second one, defined as deductive method, is founded on micromechanical concepts. Within the general framework of the deductive method, basically, two main approaches can be identified: the so-called simplified micromodelling approach and the detailed micromodelling approach.

The simplified micromodelling approach consists in modelling mortar and brick/mortar interfaces as a whole discontinuous element, usually referred to as joint. In the last few decades, after the first attempt due to Page (1978), several authors have focused on this kind of micromodelling approach and a number of models can be found in the specialised literature (Lotfi and Shing, 1994; Gambarotta and Lagomarsino, 1997); (de Buhan and de Felice, 1997; Lourenço, and Rots, 1997; Drosopoulosa, Stavroulakis and Massalasa, 2006; Milani, Lourenço and Tralli, 2006; Giambanco and Di Gati, 1997; Raous, Cangemi and Cocu, 1999; Calderini and Lagomarsino, 2008; Spada, Giambanco and Rizzo, 2009). Lotfi and Shing (1994), Lourenço, and Rots (1997), Gambarotta and Lagomarsino (1997), Giambanco and Di Gati (1997), among others, have proposed 
interface models, which include concepts developed in the theory of plasticity for nonstandard materials and in fracture mechanics. All these models adopt a Mohr-Coulomb failure criteria and use an internal variable to describe a post-peak softening behavior. These interface constitutive models are usually expressed in terms of contact tractions and conjugate generalised joint strains, derived from the discontinuities of the displacements at the joint. In particular, Lotfi and Shing (1994) presented a constitutive model for dilatant interfaces that was able to simulate initiation and propagation of fracture under combined normal and tangential stresses. Gambarotta and Lagomarsino (1997) proposed a simplified micromodelling method, based on continuum damage mechanics, aimed at taking into account both the mortar damage and the brick/mortar decohesion caused by opening and frictional sliding. Moreover, Raous, Cangemi and Cocu (1999) have developed a model, improved by Monerie and Raous (2000), which predicts the damage evolution at the interface among two initially bonded deformable bodies. They proposed a consistent model that accounts for adhesion, Coulomb friction and unilateral contact. According to Raous, Cangemi and Cocu (1999), adhesion and friction are strictly coupled with damage, which has been modelled in agreement with Fremond (1987). This kind of interface model was adopted by Fouchal, Lebon and Titeux (2009), to model mortar joints in masonry assemblies.

In the detailed micromodelling approach, the masonry joints are represented by mortar continuous elements and by discontinuous brick/mortar interface elements. This approach is less common in literature than the simplified one, probably due to its significant computational cost in numerical analysis of masonry structures. Nevertheless, it has been proven to be a powerful tool in simulating, in an accurate way, the behavior of small masonry assemblies (Alfano and Sacco, 2006; Gabor et al., 2006; Pelissou and Lebon, 2009; Rekik and Lebon, 2010, 2012; Scimemi, Giambanco and Spada, 2014). Alfano and Sacco (2006) proposed an interesting interface model, combining arguments from elastoplasticity and continuum damage mechanics, introducing, within an interface representative elementary volume, the distinction between a linear-elastic-undamaged zone and a damaged zone and considering an unilateral Coulomb friction law. Pelissou and Lebon (2009) applied the model by Gambarotta and Lagomarsino for characterizing the local behavior of the brick/mortar interface through the matched asymptotic expansion method (Lebon, Rizzoni and Ronel-Idrissi, 2014; Lebon and Rizzoni, 2010; Lebon and Zaittouni, 2010; Lebon and Rizzoni, 2011).

Within the framework of the study of behavior of brittle materials, with particular reference to masonry, the Saint Venant-Kirchhoff material has been employed by Briseghella, Pavan and Secchi (2011). In particular, they investigated the softening behavior and the crack propagation in brittle materials through a formulation in finite strains and finite displacements.

In this paper, a nonlinear-imperfect interface model is proposed. Therefore, the formulated interface law is employed to model brick/mortar interfaces in masonry structures, within the framework of the detailed micromechanical approach. The proposed model is obtained from a consolidated approach (Rekik and Lebon, 2010, 2012; Fouchal et al., 2014), also defined as Imperfect Interface Approach (IIA), coupling arguments of asymptotic analysis (Abdelmoula, Coutris and Marigo, 2011; Benveniste, 2006; Lebon and Rizzoni, 2008, 2010, 2011; Rizzoni and Lebon, 2013; Rizzoni et al., 2014) extended to the finite strain theory (Dumont, Lebon and Rizzoni, 2014; Rizzoni, Dumont and Lebon, 2015), with a homogenisation method for microcracked media under the noninteracting approximation (NIA) (Kachanov, 1994; Mauge and Kachanov, 1994; Tsukrov and Kachanov, 2000; Kachanov and Sevostianov, 2005; Sevostianov and Kachanov, 
2013). In particular, the asymptotic expansion technique has been treated by many authors, cf. Klarbring (1991) for general applications, Cecchi and Sab (2002) as far as it concerns the application to masonry and Avila-Pozos, Klarbring and Movchan (1999) for the application to an orthotropic highly inhomogeneous layered structure.

The proposed approach aims to take into account at the macroscale, some microscale effects as microcracking and locally large deformations, to reproduce the overall pre-peak response of small masonry structures. The principal peculiarities of the proposed model are the analysis of a St. Venant-Kirchhoff material (Dumont, Lebon and Rizzoni, 2014; Rizzoni, Dumont and Lebon, 2015), the introduction of microcracks at the interface level (Rekik and Lebon, 2010, 2012; Fouchal et al., 2014) and the introduction of a smooth interfacial roughness (Fouchal et al., 2014). The model proposed in this paper is expected to be useful in all those mechanical problems where solid interfaces together with geometrical and material nonlinearities have to be taken into account in a simple and reliable way at the macroscale. Potential applications could range from the bonding characterisation of cold-welded metallic joints (Zhang and Bay, 1997; Lu et al., 2010) to the collapse of FRP adhesive joints (Ascione, 2009b,a) and the simulation of masonry-FRP bond (Basilio et al., 2014; Ceroni et al., 2014), with special attention to the modelling of GFRP beams obtained by bonding simple panels (Ascione et al., 2015). In particular, its applicability in modelling brick/mortar interfaces is illustrated in Section 4.

The paper is organised as follows. In Section 2, the general theoretical background is traced, by introducing the reference problem, and the interface model is developed within the context of nonlinear elasticity by extending the asymptotic strategy proposed in Rizzoni, Dumont and Lebon (2015) to St. Venant-Kirchhoff anisotropic materials. Due to the assumption of anisotropy, additional terms have to be taken into account within the analysis (cf. coefficients $\hat{A}_{33 \alpha 3}, \hat{A}_{\beta 3 \alpha 3}, \ldots$ in Eqs. (23) and (24)). Remarkably, our calculations show that only the elastic coefficient $\hat{A}_{3333}$ enters the final contact law. This law is specialised to the case of orthotropic material in Section 3, where two model enhancements are introduced to take into account the microcracking phenomenon and to model geometrical imperfection of the interface through a smooth roughness. Finally, in Section 4, numerical results based on the proposed approach are presented and discussed. A numerical benchmark, developed in the finite element method framework, is employed to establish the model soundness and consistency. Numerical comparisons with the case of linear elasticity are also performed. In the paper, the Einstein summation rule is used, Latin indexes take the values 1, 2, 3 and Greek indexes get the values 1 and 2 .

\section{A St. Venant-Kirchhoff interface model}

\subsection{General framework}

Let an orthonormal Cartesian frame $\left(O ; \mathbf{e}_{1}, \mathbf{e}_{2}, \mathbf{e}_{3}\right)$ be introduced, with $x_{1}, x_{2}$ and $x_{3}$ be the corresponding coordinates of a particle belonging to the system $\Omega^{\varepsilon}$. Accordingly, let define the composite system $\Omega^{\varepsilon}=\Omega_{ \pm}^{\varepsilon} \cup \mathcal{B}^{\varepsilon}$ comprising two bodies $\Omega_{ \pm}^{\varepsilon}=\left\{\left(x_{1}, x_{2}, x_{3}\right) \in\right.$ $\left.\Omega^{\varepsilon}: \pm x_{3}>\frac{\varepsilon}{2}\right\}$ denoted as adherents and the thin layer $\mathcal{B}^{\varepsilon}=\left\{\left(x_{1}, x_{2}, x_{3}\right) \in \Omega^{\varepsilon}:\left|x_{3}\right|<\right.$ $\left.\frac{\varepsilon}{2}\right\}$ denoted in what follows as interphase (or adhesive), and let assume these constituents to be perfectly bonded through the surfaces $\mathcal{S}_{ \pm}^{\varepsilon}=\left\{\left(x_{1}, x_{2}, x_{3}\right) \in \Omega^{\varepsilon}: x_{3}= \pm \frac{\varepsilon}{2}\right\}$. The origin of the reference system belongs to the midplane of the interphase and the $x_{3}$-axis runs perpendicular to the open bounded set $\mathcal{S}=\left\{\left(x_{1}, x_{2}, x_{3}\right) \in \Omega^{\varepsilon}: x_{3}=0\right\}$, in the following referred as interface. The materials of the adhesive and the adherents are 
both nonlinear and modelled as St. Venant-Kirchhoff materials. On a part $\Gamma_{1}$ of the external boundary $\partial \Omega^{\varepsilon}$, a surface load $\mathbf{p}$ is applied, and on a part $\Gamma_{0}$ of $\partial \Omega^{\varepsilon}$ such that $\Gamma_{0} \cap \Gamma_{1}=$ $\emptyset$ the following boundary condition is imposed $\mathbf{u}=0$. Moreover, it is assumed that the boundary conditions are imposed far from the interphase domain: $\Gamma_{0} \cap \mathcal{B}^{\varepsilon}=\emptyset, \Gamma_{1} \cap \mathcal{B}^{\varepsilon}=$ $\emptyset$. Finally, a body force $\mathrm{f}$ is considered acting on the adherents $\Omega_{ \pm}^{\varepsilon}$.

Accordingly, the equations governing the equilibrium problem of the composite system are expressed as follows (Krasucki, Münch, and Ousset, 2001):

$$
\begin{cases}\left(s_{i j}^{\varepsilon}+s_{k j}^{\varepsilon} u_{i, k}^{\varepsilon}\right), j+f_{i}=0 & \text { in } \Omega_{ \pm}^{\varepsilon} \\ \left(s_{i j}^{\varepsilon}+s_{k j}^{\varepsilon} u_{i, k}^{\varepsilon}\right) n_{j}=p_{i} & \text { on } \Gamma_{1} \\ \left(s_{i j}^{\varepsilon}+s_{k j}^{\varepsilon} u_{i, k}^{\varepsilon}\right), j=0 & \text { in } \mathcal{B}^{\varepsilon} \\ {\left[\left[s_{i 3}^{\varepsilon}+s_{k 3}^{\varepsilon} u_{i, k}^{\varepsilon}\right]\right]=0} & \text { on } \mathcal{S}_{ \pm}^{\varepsilon} \\ \llbracket u_{i}^{\varepsilon} \rrbracket=0 & \text { on } \mathcal{S}_{ \pm}^{\varepsilon} \\ u_{i}^{\varepsilon}=0 & \text { on } \Gamma_{0} \\ s_{i j}^{\varepsilon}=A_{i j k}^{ \pm} E_{h k}\left(u^{\varepsilon}\right) & \text { in } \Omega_{ \pm}^{\varepsilon} \\ s_{i j}^{\varepsilon}=A_{i j h k}^{\varepsilon} E_{h k}\left(u^{\varepsilon}\right) & \text { in } \mathcal{B}^{\varepsilon}\end{cases}
$$

where $\mathbf{s}^{\varepsilon}$ is the second Piola-Kirchhoff stress tensor, $\mathbf{E}\left(u^{\varepsilon}\right)$ is the Green-Lagrange strain tensor $\left(E_{i j}\left(u^{\varepsilon}\right)=\frac{1}{2}\left(u_{i, j}+u_{j, i}+u_{k, i} u_{k, j}\right)\right.$ with $\left.i, j=1,2,3\right)$ and $\mathbb{A}^{ \pm}, \mathbb{A}^{\varepsilon}$ are the elasticity tensors of the deformable adherents and of the adhesive, respectively. Such an interphase material is assumed to be anisotropic. Moreover, the soft-interphase assumption holds, i.e. elasticity coefficients linearly rescaling with $\varepsilon$, according to the following relation (Geymonat, Krasucki and Lenci, 1999):

$$
A_{i j k l}^{\varepsilon}=\varepsilon \hat{A}_{i j k l}
$$

where $\hat{\mathbb{A}}$ is an anisotropic elastic tensor of the same order of magnitude of the elastic tensors $\mathbb{A}^{ \pm}$of the adherents (Geymonat, Krasucki and Lenci, 1999).

\subsection{Interface law: asymptotic expansion method}

Since the interphase is assumed to behave as a thin layer of thickness $\varepsilon$, it is natural to seek the solution of the equilibrium problem, expressed by Eqs. (1), by using asymptotic expansions with respect to the small parameter $\varepsilon$ (Lebon, Rizzoni and Ronel-Idrissi, 2014). In particular, the following asymptotic series with fractional powers are exploited:

$$
\left\{\begin{array}{l}
\mathbf{u}^{\varepsilon}\left(x_{1}, x_{2}, x_{3}\right)=\mathbf{u}^{0}+\varepsilon^{1 / 3} \mathbf{u}^{1}+\varepsilon^{2 / 3} \mathbf{u}^{2}+\varepsilon \mathbf{u}^{3}+\varepsilon^{4 / 3} \mathbf{u}^{4}+\varepsilon^{5 / 3} \mathbf{u}^{5}+\varepsilon^{2} \mathbf{u}^{6}+o\left(\varepsilon^{2}\right) \\
\mathbf{s}^{\varepsilon}\left(x_{1}, x_{2}, x_{3}\right)=\mathbf{s}^{0}+\varepsilon^{1 / 3} \mathbf{s}^{1}+\varepsilon^{2 / 3} \mathbf{s}^{2}+\varepsilon \mathbf{s}^{3}+\varepsilon^{4 / 3} \mathbf{s}^{4}+\varepsilon^{5 / 3} \mathbf{s}^{5}+\varepsilon^{2} \mathbf{s}^{6}+o\left(\varepsilon^{2}\right)
\end{array}\right.
$$

It is worth remarking that such a choice of a fractional expansion is due to energy-based evidences (Rizzoni, Dumont and Lebon, 2015). In particular, from a quite simple monodimensional example, proposed in Rizzoni, Dumont and Lebon (2015), it has been put in evidence that the solution in terms of displacement jump is proportional to $\varepsilon^{\frac{2}{3}}$.

In agreement with Ciarlet (1997), let the change of variable $\hat{\mathrm{g}}:\left(x_{1}, x_{2}, x_{3}\right) \rightarrow$ $\left(z_{1}, z_{2}, z_{3}\right)$ be introduced in $\mathcal{B}^{\varepsilon}$, with $z_{1}=x_{1}, z_{2}=x_{2}, z_{3}=x_{3} / \varepsilon$. Moreover, let the change of variable $\overline{\mathrm{g}}:\left(x_{1}, x_{2}, x_{3}\right) \rightarrow\left(z_{1}, z_{2}, z_{3}\right)$ be introduced in $\Omega_{ \pm}^{\varepsilon}$, with $z_{1}=x_{1}$, $z_{2}=x_{2}, z_{3}=x_{3} \pm(1-\varepsilon) / 2$. As a result, the interphase $\mathcal{B}^{\varepsilon}$ and the adherents $\Omega_{ \pm}^{\varepsilon}$ are scaled in domains of unitary thickness $\mathcal{B}$ and $\Omega_{ \pm}$, respectively. In what follows, symbols $\cdot$ and $\hat{\imath}$ refer to rescaled quantities for $\mathcal{B}$ and $\Omega_{ \pm}$, respectively. More precisely, $\hat{\mathbf{u}}^{\varepsilon}=\mathbf{u}^{\varepsilon} \circ \hat{\mathbf{g}}^{-1}$ and $\hat{\mathbf{s}}^{\varepsilon}=\mathbf{s}^{\varepsilon} \circ \hat{\mathbf{g}}^{-1}$ denote displacement and stress fields for $\mathcal{B}$, and $\overline{\mathbf{u}}^{\varepsilon}=$ 
$\mathbf{u}^{\varepsilon} \circ \overline{\mathbf{g}}^{-1}$ and $\overline{\mathbf{s}}^{\varepsilon}=\mathbf{s}^{\varepsilon} \circ \overline{\mathbf{g}}^{-1}$ are displacement vector and stress tensor for $\Omega_{ \pm}, \mathbf{u}^{\varepsilon}$ and $\mathbf{s}^{\varepsilon}$ being the corresponding fields on the system $\Omega^{\varepsilon}$. The internal and external forces, $\mathbf{f}$ and $\mathbf{p}$, respectively, are assumed to be independent of $\varepsilon$. As a consequence, it is set $\overline{\mathbf{f}}\left(z_{1}, z_{2}, z_{3}\right)=\mathbf{f}\left(x_{1}, x_{2}, x_{3}\right)$ and $\overline{\mathbf{p}}\left(z_{1}, z_{2}, z_{3}\right)=\mathbf{p}\left(x_{1}, x_{2}, x_{3}\right)$. Moreover, under the change of variables, the domains $\Gamma_{0}$ and $\Gamma_{1}$ are transformed into the domains denoted by $\bar{\Gamma}_{0}$ and $\bar{\Gamma}_{1}$, respectively. As a result, the governing equations of the equilibrium problem, in the rescaled composite system, are expressed as follows:

$$
\begin{cases}\left(\bar{s}_{i j}+\bar{s}_{k j} \bar{u}_{i, k}\right)_{, j}+\bar{f}_{i}=0 & \text { in } \Omega_{ \pm} \\ \left(\bar{s}_{i j}+\bar{s}_{k j} \bar{u}_{i, k}\right) n_{j}=\bar{p}_{i} & \text { on } \bar{\Gamma}_{1} \\ \left(\hat{s}_{i \alpha}+\hat{s}_{k \alpha} \hat{u}_{i, k}\right)_{, \alpha}+\frac{1}{\varepsilon}\left(\hat{s}_{i 3}+\hat{s}_{k 3} \hat{u}_{i, k}\right)_{, 3}=0 & \text { in } \mathcal{B} \\ \bar{s}_{i 3}+\bar{s}_{k 3} \bar{u}_{i, k}=\hat{s}_{i 3}+\hat{s}_{\alpha 3} \hat{u}_{i, \alpha}+\frac{1}{\varepsilon} \hat{s}_{33} \hat{u}_{i, 3} & \text { on } \mathcal{S}_{ \pm} \\ \bar{u}_{i}=\hat{u}_{i} & \text { on } \mathcal{S}_{ \pm} \\ \bar{u}_{i}=0 & \text { on } \bar{\Gamma}_{0} \\ \bar{s}_{i j}=A_{i j h k}^{ \pm} \bar{E}_{h k}(\bar{u}) & \text { in } \Omega_{ \pm} \\ \hat{s}_{i j}=A_{i j h k}^{\varepsilon} \hat{E}_{h k}(\hat{u}) & \text { in } \mathcal{B}\end{cases}
$$

where $\overline{\mathbf{E}}, \hat{\mathbf{E}}$ denote the rescaled Green-Lagrange strain tensors in the adherents and in the adhesive.

Based on Eqs. (3) the relevant fields, in the rescaled adhesive and adherents, can be expressed as asymptotic expansions in the following way:

$$
\left\{\begin{array}{l}
\hat{\mathbf{s}}^{\varepsilon}\left(z_{1}, z_{2}, z_{3}\right)=\hat{\mathbf{s}}^{0}+\varepsilon^{1 / 3} \hat{\mathbf{s}}^{1}+\varepsilon^{2 / 3} \hat{\mathbf{s}}^{2}+\varepsilon \hat{\mathbf{s}}^{3}+\varepsilon^{4 / 3} \hat{\mathbf{s}}^{4}+\varepsilon^{5 / 3} \hat{\mathbf{s}}^{5}+\varepsilon^{2} \hat{\mathbf{s}}^{6}+o\left(\varepsilon^{2}\right) \\
\overline{\mathbf{s}}^{\varepsilon}\left(z_{1}, z_{2}, z_{3}\right)=\overline{\mathbf{s}}^{0}+\varepsilon^{1 / 3} \overline{\mathbf{s}}^{1}+\varepsilon^{2 / 3} \overline{\mathbf{s}}^{2}+\varepsilon \overline{\mathbf{s}}^{3}+\varepsilon^{4 / 3} \overline{\mathbf{s}}^{4}+\varepsilon^{5 / 3} \overline{\mathbf{s}}^{5}+\varepsilon^{2} \overline{\mathbf{s}}^{6}+o\left(\varepsilon^{2}\right) \\
\hat{\mathbf{u}}^{\varepsilon}\left(z_{1}, z_{2}, z_{3}\right)=\hat{\mathbf{u}}^{0}+\varepsilon^{1 / 3} \hat{\mathbf{u}}^{1}+\varepsilon^{2 / 3} \hat{\mathbf{u}}^{2}+\varepsilon \hat{\mathbf{u}}^{3}+\varepsilon^{4 / 3} \hat{\mathbf{u}}^{4}+\varepsilon^{5 / 3} \hat{\mathbf{u}}^{5}+\varepsilon^{2} \hat{\mathbf{u}}^{6}+o\left(\varepsilon^{2}\right) \\
\overline{\mathbf{u}}^{\varepsilon}\left(z_{1}, z_{2}, z_{3}\right)=\overline{\mathbf{u}}^{0}+\varepsilon^{1 / 3} \overline{\mathbf{u}}^{1}+\varepsilon^{2 / 3} \overline{\mathbf{u}}^{2}+\varepsilon \overline{\mathbf{u}}^{3}+\varepsilon^{4 / 3} \overline{\mathbf{u}}^{4}+\varepsilon^{5 / 3} \overline{\mathbf{u}}^{5}+\varepsilon^{2} \overline{\mathbf{u}}^{6}+o\left(\varepsilon^{2}\right)
\end{array}\right.
$$

In the following, the conditions holding in the rescaled interphase $\mathcal{B}$ are detailed. These latter are obtained by substituting the first of Eqs. (5) into the equilibrium equation holding in the interphase (i.e., third equation of the system (4)) and by identifying similar terms with respect to the power of the parameter $\varepsilon$ :

- $\quad$ Power of $\varepsilon:-2$

$$
\left(\hat{u}_{i, 3}^{0} \hat{s}_{33}^{0}\right)_{, 3}=0
$$

- $\quad$ Power of $\varepsilon:-5 / 3$

$$
\left(\hat{u}_{i, 3}^{0} \hat{s}_{33}^{1}+\hat{u}_{i, 3}^{1} \hat{s}_{33}^{0}\right)_{, 3}=0,
$$

- $\quad$ Power of $\varepsilon:-4 / 3$

$$
\left(\hat{u}_{i, 3}^{0} \hat{s}_{33}^{2}+\hat{u}_{i, 3}^{1} \hat{s}_{33}^{1}+\hat{u}_{i, 3}^{2} \hat{s}_{33}^{0}\right)_{, 3}=0,
$$

- Power of $\varepsilon:-1$

$$
\begin{aligned}
& \left(\hat{u}_{i, 3}^{0} \hat{s}_{3 \alpha}^{0}\right)_{, \alpha}+\left(\hat{s}_{i 3}^{0}+\hat{u}_{i, \alpha}^{0} \hat{s}_{3 \alpha}^{0}\right)_{, 3} \\
& +\left(\hat{u}_{i, 3}^{0} \hat{s}_{33}^{3}+\hat{u}_{i, 3}^{1} \hat{s}_{33}^{2}+\hat{u}_{i, 3}^{2} \hat{s}_{33}^{1}+\hat{u}_{i, 3}^{3} \hat{s}_{33}^{0}\right)_{, 3}=0,
\end{aligned}
$$


- Power of $\varepsilon:-2 / 3$

$$
\begin{aligned}
& \left(\hat{u}_{i, 3}^{0} \hat{s}_{3 \alpha}^{1}+\hat{u}_{i, 3}^{1} \hat{s}_{3 \alpha}^{0}\right)_{, \alpha}+\left(\hat{s}_{i 3}^{1}+\hat{u}_{i, \alpha}^{0} \hat{s}_{3 \alpha}^{1}+\hat{u}_{i, \alpha}^{1} \hat{s}_{3 \alpha}^{0}\right)_{, 3} \\
& +\left(\hat{u}_{i, 3}^{0} \hat{s}_{33}^{4}+\hat{u}_{i, 3}^{1} \hat{s}_{33}^{3}+\hat{u}_{i, 3}^{2} \hat{s}_{33}^{2}+\hat{u}_{i, 3}^{3} \hat{s}_{33}^{1}+\hat{u}_{i, 3}^{4} \hat{s}_{33}^{0}\right)_{, 3}=0,
\end{aligned}
$$

- Power of $\varepsilon:-1 / 3$

$$
\begin{aligned}
& \left(\hat{u}_{i, 3}^{0} \hat{s}_{3 \alpha}^{2}+\hat{u}_{i, 3}^{1} \hat{s}_{3 \alpha}^{1}+\hat{u}_{i, 3}^{2} \hat{s}_{3 \alpha}^{0}\right)_{, \alpha} \\
& +\left(\hat{s}_{i 3}^{2}+\hat{u}_{i, \alpha}^{0} \hat{s}_{3 \alpha}^{2}+\hat{u}_{i, \alpha}^{1} \hat{s}_{3 \alpha}^{1}+\hat{u}_{i, \alpha}^{2} \hat{s}_{3 \alpha}^{0}\right)_{, 3} \\
& +\left(\hat{u}_{i, 3}^{0} \hat{s}_{33}^{5}+\hat{u}_{i, 3}^{1} \hat{s}_{33}^{4}+\hat{u}_{i, 3}^{2} \hat{s}_{33}^{3}+\hat{u}_{i, 3}^{3} \hat{s}_{33}^{2}+\hat{u}_{i, 3}^{4} \hat{s}_{33}^{1}+\hat{u}_{i, 3}^{5} \hat{s}_{33}^{0}\right)_{, 3}=0,
\end{aligned}
$$

- Power of $\varepsilon: 0$

$$
\begin{aligned}
& \left(\hat{u}_{i, 3}^{0} \hat{s}_{3 \alpha}^{3}+\hat{u}_{i, 3}^{1} \hat{s}_{3 \alpha}^{2}+\hat{u}_{i, 3}^{2} \hat{s}_{3 \alpha}^{1}+\hat{u}_{i, 3}^{3} \hat{s}_{3 \alpha}^{0}\right)_{, \alpha}+\left(\hat{s}_{i \alpha}^{0}+\hat{s}_{\alpha \beta}^{0} \hat{u}_{i, \beta}^{0}\right)_{\alpha} \\
& +\left(\hat{s}_{i 3}^{3}+\hat{u}_{i, \alpha}^{0} \hat{s}_{3 \alpha}^{3}+\hat{u}_{i, \alpha}^{1} \hat{s}_{3 \alpha}^{2}+\hat{u}_{i, \alpha}^{2} \hat{s}_{3 \alpha}^{1}+\hat{u}_{i, \alpha}^{3} \hat{s}_{3 \alpha}^{0}\right)_{, 3} \\
& +\left(\hat{u}_{i, 3}^{0} \hat{s}_{33}^{6}+\hat{u}_{i, 3}^{1} \hat{s}_{33}^{5}+\hat{u}_{i, 3}^{2} \hat{s}_{33}^{4}+\hat{u}_{i, 3}^{3} \hat{s}_{33}^{3}+\hat{u}_{i, 3}^{4} \hat{s}_{33}^{2}+\hat{u}_{i, 3}^{5} \hat{s}_{33}^{1}+\hat{u}_{i, 3}^{6} \hat{s}_{33}^{0}\right)_{, 3}=0,
\end{aligned}
$$

By substituting the first two equations of (5) into the continuity condition of the traction vector holding through the rescaled interfaces $\mathcal{S}_{ \pm}$(i.e., fourth equation of system (4)), and by applying the usual identification procedure, the following relationships are obtained:

- $\quad$ Power of $\varepsilon:-1$

$$
0=\left(\hat{u}_{i, 3}^{0} \hat{s}_{33}^{0}\right)
$$

- $\quad$ Power of $\varepsilon:-2 / 3$

$$
0=\left(\hat{u}_{i, 3}^{0} \hat{s}_{33}^{1}+\hat{u}_{i, 3}^{1} \hat{s}_{33}^{0}\right)
$$

- $\quad$ Power of $\varepsilon:-1 / 3$

$$
0=\left(\hat{u}_{i, 3}^{0} \hat{s}_{33}^{2}+\hat{u}_{i, 3}^{1} \hat{s}_{33}^{1}+\hat{u}_{i, 3}^{2} \hat{s}_{33}^{0}\right)
$$

- $\quad$ Power of $\varepsilon: 0$

$$
\left(\bar{s}_{i 3}^{0}+\bar{u}_{i k}^{0} \bar{s}_{k 3}^{0}\right)=\left(\hat{s}_{i 3}^{0}+\hat{u}_{i, \alpha}^{0} \hat{s}_{\alpha 3}^{0}+\hat{u}_{i, 3}^{0} \hat{s}_{33}^{3}+\hat{u}_{i, 3}^{1} \hat{s}_{33}^{2}+\hat{u}_{i, 3}^{2} \hat{s}_{33}^{1}+\hat{u}_{i, 3}^{3} \hat{s}_{33}^{0}\right)
$$

- $\quad$ Power of $\varepsilon: 1 / 3$

$$
\begin{aligned}
& \left(\bar{s}_{i 3}^{1}+\bar{u}_{i, k}^{0} \bar{s}_{k 3}^{1}+\bar{u}_{i, k}^{1} \bar{s}_{k 3}^{0}\right)=\left(\hat{s}_{i 3}^{1}+\hat{u}_{i, \alpha}^{0} \hat{s}_{\alpha 3}^{1}+\hat{u}_{i, \alpha}^{1} \hat{s}_{\alpha 3}^{0}\right) \\
& +\left(\hat{u}_{i, 3}^{0} \hat{s}_{33}^{4}+\hat{u}_{i, 3}^{1} \hat{s}_{33}^{3}+\hat{u}_{i, 3}^{2} \hat{s}_{33}^{2}+\hat{u}_{i, 3}^{3} \hat{s}_{33}^{1}+\hat{u}_{i, 3}^{4} \hat{s}_{33}^{0}\right)
\end{aligned}
$$


- $\quad$ Power of $\varepsilon: 2 / 3$

$$
\begin{aligned}
& \left(\bar{s}_{i 3}^{2}+\bar{u}_{i, k}^{0} \bar{s}_{k 3}^{2}+\bar{u}_{i, k}^{1} \bar{s}_{k 3}^{1}+\bar{u}_{i, k}^{2} \bar{s}_{k 3}^{0}\right)=\left(\hat{s}_{33}^{2}+\hat{u}_{i, \alpha}^{0} \hat{s}_{\alpha 3}^{2}+\hat{u}_{i, \alpha}^{1} \hat{s}_{\alpha 3}^{1}+\hat{u}_{i, \alpha}^{0} \hat{s}_{\alpha 3}^{2}\right) \\
& +\left(\hat{u}_{i, 3}^{0} \hat{s}_{33}^{5}+\hat{u}_{i, 3}^{1} \hat{s}_{33}^{4}+\hat{u}_{i, 3}^{2} \hat{s}_{33}^{3}+\hat{u}_{i, 3}^{3} \hat{s}_{33}^{2}+\hat{u}_{i, 3}^{4} \hat{s}_{33}^{1}+\hat{u}_{i, 3}^{5} \hat{s}_{33}^{0}\right)
\end{aligned}
$$

- $\quad$ Power of $\varepsilon: 1$

$$
\begin{aligned}
& \left(\bar{s}_{i 3}^{3}+\bar{u}_{i, k}^{0} \bar{s}_{k 3}^{3}+\bar{u}_{i, k}^{1} \bar{s}_{k 3}^{2}+\bar{u}_{i, k}^{2} \bar{s}_{k 3}^{1}+\bar{u}_{i, k}^{3} \bar{s}_{k 3}^{0}\right) \\
& =\left(\hat{s}_{i 3}^{3}+\hat{u}_{i, \alpha}^{0} \hat{s}_{\alpha 3}^{3}+\hat{u}_{i, \alpha}^{1} \hat{s}_{\alpha 3}^{2}+\hat{u}_{i, \alpha}^{2} \hat{s}_{\alpha 3}^{1}+\hat{u}_{i, \alpha}^{3} \hat{s}_{\alpha 3}^{0}\right) \\
& +\left(\hat{u}_{i, 3}^{0} \hat{s}_{33}^{6}+\hat{u}_{i, 3}^{1} \hat{s}_{33}^{5}+\hat{u}_{i, 3}^{2} \hat{s}_{33}^{4}+\hat{u}_{i, 3}^{3} \hat{s}_{33}^{3}+\hat{u}_{i, 3}^{4} \hat{s}_{33}^{2}+\hat{u}_{i, 3}^{5} \hat{s}_{33}^{1}+\hat{u}_{i, 3}^{6} \hat{s}_{33}^{0}\right)
\end{aligned}
$$

It is worth noting that the above equations hold both in $\mathcal{S}_{+}$and in $\mathcal{S}_{-}$. For the sake of brevity, they have been detailed only in one case. Moreover, by remarking that the lefthand sides in Eqs. (16-19) can be identified as the expansions of the $\mathbf{e}_{3}$ components of the first Piola-Kirchhoff stress tensor $\bar{P}_{i 3}=\left(\bar{s}_{i 3}+\bar{u}_{i k} \bar{s}_{k 3}\right)$ in the adherents, a significant simplification of these equations it is possible.

According to the soft-material-interphase assumption, by substituting Eq. (2) in the constitutive law holding in the interphase $\mathcal{B}$ of the rescaled domain (i.e., last equation of the problem (4)), written for $j=3$, the following conditions are deduced:

- $\quad$ Power of $\varepsilon:-1$

$$
0=\left(\hat{u}_{k, 3}^{0} \hat{u}_{k, 3}^{0}\right)
$$

- $\quad$ Power of $\varepsilon:-2 / 3$

$$
0=\left(\hat{u}_{k, 3}^{0} \hat{u}_{k, 3}^{1}+\hat{u}_{k, 3}^{1} \hat{u}_{k, 3}^{0}\right)
$$

- $\quad$ Power of $\varepsilon:-1 / 3$

$$
0=\left(\hat{u}_{k, 3}^{0} \hat{u}_{k, 3}^{2}+\hat{u}_{k, 3}^{1} \hat{u}_{k, 3}^{1}+\hat{u}_{k, 3}^{2} \hat{u}_{k, 3}^{0}\right)
$$

- Power of $\varepsilon$ : 0

$$
\begin{aligned}
& \hat{s}_{\alpha 3}^{0}=\hat{A}_{33 \alpha 3}\left[\hat{u}_{3,3}^{0}+\left(\hat{u}_{s, 3}^{0} \hat{u}_{s, 3}^{3}+\hat{u}_{s, 3}^{1} \hat{u}_{s, 3}^{2}\right)\right]+\frac{1}{2} \hat{A}_{\beta 3 \alpha 3}\left(\hat{u}_{\beta, 3}^{0}+\hat{u}_{s, \beta}^{0} \hat{u}_{s, 3}^{0}\right) \\
& \hat{s}_{33}^{0}=\hat{A}_{3333}\left[\hat{u}_{3,3}^{0}+\left(\hat{u}_{s, 3}^{0} \hat{u}_{s, 3}^{3}+\hat{u}_{s, 3}^{1} \hat{u}_{s, 3}^{2}\right)\right]+\frac{1}{2} \hat{A}_{33 \beta 3}\left(\hat{u}_{\beta, 3}^{0}+\hat{u}_{s, \beta}^{0} \hat{u}_{s, 3}^{0}\right)
\end{aligned}
$$

- Power of $\varepsilon: 1 / 3$

$$
\begin{aligned}
\hat{s}_{\alpha 3}^{1}= & \hat{A}_{33 \alpha 3}\left[\hat{u}_{3,3}^{1}+\left(\hat{u}_{s, 3}^{0} \hat{u}_{s, 3}^{4}+\hat{u}_{s, 3}^{1} \hat{u}_{s, 3}^{3}+\frac{1}{2} \hat{u}_{s, 3}^{2} \hat{u}_{s, 3}^{2}\right)\right] \\
& +\frac{1}{2} \hat{A}_{\beta 3 \alpha 3}\left(\hat{u}_{\beta, 3}^{1}+\hat{u}_{s, \beta}^{0} \hat{u}_{s, 3}^{1}+\hat{u}_{s, \beta}^{1} \hat{u}_{s, 3}^{0}\right) \\
\hat{s}_{33}^{1}= & \hat{A}_{3333}\left[\hat{u}_{3,3}^{1}+\left(\hat{u}_{s, 3}^{0} \hat{u}_{s, 3}^{4}+\hat{u}_{s, 3}^{1} \hat{u}_{s, 3}^{3}+\frac{1}{2} \hat{u}_{s, 3}^{2} \hat{u}_{s, 3}^{2}\right)\right] \\
& +\frac{1}{2} \hat{A}_{33 \beta 3}\left(\hat{u}_{\beta, 3}^{1}+\hat{u}_{s, \beta}^{0} \hat{u}_{s, 3}^{1}+\hat{u}_{s, \beta}^{1} \hat{u}_{s, 3}^{0}\right)
\end{aligned}
$$


From Eqs. (20-23) it follows that:

$$
\begin{aligned}
& \hat{\mathbf{u}}_{, 3}^{0}=0 \text { in } \mathcal{B} \Rightarrow\left[\hat{\mathbf{u}}^{0}\right]=0 \\
& \hat{\mathbf{u}}_{, 3}^{1}=0 \text { in } \mathcal{B} \Rightarrow\left[\hat{\mathbf{u}}^{1}\right]=0 \\
& \hat{s}_{\alpha 3}^{0}=0=\hat{s}_{33}^{0} \text { in } \mathcal{B}
\end{aligned}
$$

where it is set $[g]\left(z_{1}, z_{2}\right)=g\left(z_{1}, z_{2}, 1 / 2\right)-g\left(z_{1}, z_{2},-1 / 2\right)$ for $g: \mathcal{B} \mapsto \mathbb{R}^{3}$. By combining Eqs. (25-27) into Eqs. (6-9), the following relationship is obtained:

$$
\left(\hat{u}_{i, 3}^{2} \hat{s}_{33}^{1}\right)_{3}=0 \text { in } \mathcal{B}
$$

which integrated with respect to $z_{3}$ gives

$$
\hat{u}_{i, 3}^{2} \hat{s}_{33}^{1}=\text { const. }=\left.\bar{P}_{i 3}^{0}\right|_{\mathcal{S}_{ \pm}} \text {in } \mathcal{B}
$$

where $\left.\bar{P}_{i 3}^{0}\right|_{\mathcal{S}_{ \pm}}$is the common value taken at the interfaces $\mathcal{S}_{ \pm}$(cfr. Eq. (16)). Moreover, by substituting Eq. (24) and Eqs. (25-27) into Eq. (29) the following relationship is obtained:

$$
\frac{1}{2} \hat{A}_{3333}\left(\left|\hat{u}_{i, 3}^{2}\right|^{2} \hat{u}_{i, 3}^{2}\right)=\bar{P}_{i 3}^{0} \text { in } \mathcal{B}
$$

By solving with respect to $\hat{\mathbf{u}}_{3,}^{2}$ and by integrating with respect to $z_{3}$ one has:

$$
\left[\hat{\mathbf{u}}^{2}\right]=\frac{1}{\left(\frac{1}{2} \hat{A}_{3333}\right)^{1 / 3}} \frac{1}{\left|\overline{\mathbf{P}}^{0} \mathbf{e}_{3}\right|^{2 / 3}} \overline{\mathbf{P}}^{0} \mathbf{e}_{3}
$$

Thereby, by substituting Eqs. (20-23) into Eqs. (16-18) it is obtained that:

$$
\begin{aligned}
{\left[\overline{\mathbf{P}}^{0} \mathbf{e}_{3}\right] } & =0 \\
{\left[\overline{\mathbf{P}}^{1} \mathbf{e}_{3}\right] } & =0 \\
{\left[\overline{\mathbf{P}}^{2} \mathbf{e}_{3}\right] } & =0
\end{aligned}
$$

with

$$
\begin{aligned}
\bar{P}_{i 3}^{0} & =\left(\bar{s}_{i 3}^{0}+\bar{u}_{i, k}^{0} \bar{s}_{k 3}^{0}\right) \\
\bar{P}_{i 3}^{1} & =\left(\bar{s}_{i 3}^{1}+\bar{u}_{i, k}^{0} \bar{s}_{k 3}^{1}+\bar{u}_{i, k}^{1} \bar{s}_{k 3}^{0}\right) \\
\bar{P}_{i 3}^{2} & =\left(\bar{s}_{i 3}^{2}+\bar{u}_{i, k}^{0} \bar{s}_{k 3}^{2}+\bar{u}_{i, k}^{1} \bar{s}_{k 3}^{1}+\bar{u}_{i, k}^{2} \bar{s}_{k 3}^{0}\right)
\end{aligned}
$$

The final step of the asymptotic expansion method consists in applying some matching conditions to find the proper interface law for the limit equilibrium problem, in which the interphase is replaced by the limit interface $\mathcal{S}$ and the adherents by the domains $\Omega_{+}^{0}=$ $\left\{\left(x_{1}, x_{2}, x_{3}\right) \in \Omega: \pm x_{3}>0\right\}$. By taking into account the asymptotic expansion of the 
displacement field (3) and assuming that $\mathbf{u}^{\varepsilon}$ in the adherent can be expanded in a Taylor series representation along the $x_{3}$-direction, it results:

$$
\begin{aligned}
& \mathbf{u}^{\varepsilon}\left(\overline{\mathbf{x}}, \pm \frac{\varepsilon}{2}\right)= \mathbf{u}^{\varepsilon}\left(\overline{\mathbf{x}}, 0^{ \pm}\right) \pm \\
&= \frac{\varepsilon}{2} \mathbf{u}_{, 3}^{\varepsilon}\left(\overline{\mathbf{x}}, 0^{ \pm}\right)+\cdots \\
& \mathbf{u}^{0}\left(\overline{\mathbf{x}}, 0^{ \pm}\right)+\varepsilon^{1 / 3} \mathbf{u}^{1}\left(\overline{\mathbf{x}}, 0^{ \pm}\right)+\varepsilon^{2 / 3} \mathbf{u}^{2}\left(\overline{\mathbf{x}}, 0^{ \pm}\right) \\
& \quad+\varepsilon\left(\mathbf{u}^{3}\left(\overline{\mathbf{x}}, 0^{ \pm}\right) \pm \frac{1}{2} \mathbf{u}_{, 3}^{0}\left(\overline{\mathbf{x}}, 0^{ \pm}\right)\right)+\cdots
\end{aligned}
$$

In view of the continuity of the displacements at the interfaces $\mathcal{S}_{ \pm}^{\varepsilon}$ and $\mathcal{S}_{ \pm}$one has

$$
\begin{aligned}
& \mathbf{u}^{0}\left(\overline{\mathbf{x}}, 0^{ \pm}\right)+\varepsilon^{1 / 3} \mathbf{u}^{1}\left(\overline{\mathbf{x}}, 0^{ \pm}\right)+\varepsilon^{2 / 3} \mathbf{u}^{2}\left(\overline{\mathbf{x}}, 0^{ \pm}\right)+\cdots \\
& =\hat{\mathbf{u}}^{0}\left(\overline{\mathbf{z}}, \pm \frac{1}{2}\right)+\varepsilon^{1 / 3} \hat{\mathbf{u}}^{1}\left(\overline{\mathbf{z}}, \pm \frac{1}{2}\right)+\cdots \\
& =\overline{\mathbf{u}}^{0}\left(\overline{\mathbf{z}}, \pm \frac{1}{2}\right)+\varepsilon^{1 / 3} \overline{\mathbf{u}}^{1}\left(\overline{\mathbf{z}}, \pm \frac{1}{2}\right)+\cdots
\end{aligned}
$$

and, identifying the terms in the same powers of $\varepsilon$, it is deduced that:

$$
\begin{aligned}
& \mathbf{u}^{0}\left(\overline{\mathbf{x}}, 0^{ \pm}\right)=\hat{\mathbf{u}}^{0}\left(\overline{\mathbf{z}}, \pm \frac{1}{2}\right)=\overline{\mathbf{u}}^{0}\left(\overline{\mathbf{z}}, \pm \frac{1}{2}\right) \\
& \mathbf{u}^{1}\left(\overline{\mathbf{x}}, 0^{ \pm}\right)=\hat{\mathbf{u}}^{1}\left(\overline{\mathbf{z}}, \pm \frac{1}{2}\right)=\overline{\mathbf{u}}^{1}\left(\overline{\mathbf{z}}, \pm \frac{1}{2}\right) \\
& \mathbf{u}^{2}\left(\overline{\mathbf{x}}, 0^{ \pm}\right)=\hat{\mathbf{u}}^{2}\left(\overline{\mathbf{z}}, \pm \frac{1}{2}\right)=\overline{\mathbf{u}}^{2}\left(\overline{\mathbf{z}}, \pm \frac{1}{2}\right)
\end{aligned}
$$

Analogous results can be obtained for the tractions vector, herein expressed in terms of the first Piola-Kirchhoff tensor:

$$
\begin{aligned}
\mathbf{P}^{0}\left(\overline{\mathbf{x}}, 0^{ \pm}\right) \mathbf{e}_{3} & =\hat{\mathbf{P}}^{0}\left(\overline{\mathbf{z}}, \pm \frac{1}{2}\right) \mathbf{e}_{3}=\overline{\mathbf{P}}^{0}\left(\overline{\mathbf{z}}, \pm \frac{1}{2}\right) \mathbf{e}_{3} \\
\mathbf{P}^{1}\left(\overline{\mathbf{x}}, 0^{ \pm}\right) \mathbf{e}_{3} & =\hat{\mathbf{P}}^{1}\left(\overline{\mathbf{z}}, \pm \frac{1}{2}\right) \mathbf{e}_{3}=\overline{\mathbf{P}}^{1}\left(\overline{\mathbf{z}}, \pm \frac{1}{2}\right) \mathbf{e}_{3} \\
\mathbf{P}^{2}\left(\overline{\mathbf{x}}, 0^{ \pm}\right) \mathbf{e}_{3} & =\hat{\mathbf{P}}^{2}\left(\overline{\mathbf{z}}, \pm \frac{1}{2}\right) \mathbf{e}_{3}=\overline{\mathbf{P}}^{2}\left(\overline{\mathbf{z}}, \pm \frac{1}{2}\right) \mathbf{e}_{3}
\end{aligned}
$$

Let the following notation be adopted: $\llbracket \mathbf{g} \rrbracket:=\mathbf{g}\left(\mathbf{x}, 0^{+}\right)-\mathbf{g}\left(\mathbf{x}, 0^{-}\right)$with $\mathbf{g}: \Omega_{+}^{0} \cup \Omega_{-}^{0} \mapsto$ $\mathbb{R}^{3}$; accordingly, the proper contact conditions for the limit equilibrium problem, i.e. expressed in terms of the relevant fields defined on $\Omega_{+}^{0} \cup \Omega_{-}^{0}$, can be obtained by using this relation into the interphase laws (Eqs. (25), (26), (31) and Eqs. (32)-(34)):

$$
\begin{aligned}
{\left[\overline{\mathbf{u}}^{l}\right] } & =\left[\left[\mathbf{u}^{l}\right]\right] \quad l=0,1,2 \\
{\left[\overline{\mathbf{P}}^{l} \mathbf{e}_{3}\right] } & =\left[\left[\mathbf{P}^{l} \mathbf{e}_{3}\right]\right] \quad l=0,1,2
\end{aligned}
$$


By applying the matching relations (42), the transmission conditions for the soft interphase can be rewritten in the limit configuration $\Omega_{+}^{0} \cup \Omega_{-}^{0} \cup \mathcal{S}$ in a form involving only the fields in the adherents:

$$
\begin{aligned}
{\left[\left[\mathbf{u}^{0}\right]\right] } & =0 & & {\left[\left[\mathbf{P}^{0} \mathbf{e}_{3}\right]\right]=0 } \\
{\left[\left[\mathbf{u}^{1}\right]\right] } & =0 & {\left[\left[\mathbf{P}^{1} \mathbf{e}_{3}\right]\right] } & =0 \\
{\left[\left[\mathbf{u}^{2}\right]\right] } & =\frac{1}{\left(\frac{1}{2} \hat{A}_{3333}\right)^{1 / 3}} \frac{1}{\left|\mathbf{P}^{0} \mathbf{e}_{3}\right|^{2 / 3}} \mathbf{P}^{0} \mathbf{e}_{3} & & {\left.\left[\mathbf{P}^{2} \mathbf{e}_{3}\right]\right]=0 }
\end{aligned}
$$

which are the final expressions of the interface conditions for the proposed St. VenantKirchhoff anisotropic model. It is worth remarking that the imperfect interface condition, prescribing a jump of the displacement, appears at the second order (Eq. (45)). By recalling Eq. (2), and by taking into account the expansions (3) and the relations (35)-(37), one finds:

$$
\begin{aligned}
\mathbf{P}^{\varepsilon} \mathbf{e}_{3} & =\mathbf{P}^{0} \mathbf{e}_{3}+O\left(\varepsilon^{1 / 3}\right) \\
\llbracket \mathbf{P}^{\varepsilon} \mathbf{e}_{3} \rrbracket & =\varepsilon^{2 / 3}\left[\left[\mathbf{P}^{2} \mathbf{e}_{3}\right]\right]+O(\varepsilon) \\
\llbracket \mathbf{u}^{\varepsilon} \rrbracket & \left.=\varepsilon^{2 / 3} \llbracket\left[\mathbf{u}^{2}\right]\right]+O(\varepsilon)
\end{aligned}
$$

which, substituted into (43)-(45), give

$$
\begin{aligned}
& \llbracket \mathbf{P}^{\varepsilon} \mathbf{e}_{3} \rrbracket=0+o(\varepsilon) \\
& \mathbf{P}^{\varepsilon} \mathbf{e}_{3}=\frac{A_{3333}^{\varepsilon}}{2 \varepsilon^{3}}\left|\llbracket \mathbf{u}^{\varepsilon} \rrbracket\right|^{2} \llbracket \mathbf{u}^{\varepsilon} \rrbracket+o\left(\varepsilon^{1 / 3}\right)
\end{aligned}
$$

Eqns. $(49,50)$ are the relevant interface law, crucial from a computational point of view, and in the following adopted within the numerical validation.

\section{Model enhancements}

In this section, two model enhancements are proposed and detailed. The first concerns the introduction of the damage, particularly microcracking, in the constitutive properties of the orthotropic interphase. The second one consists in adding an interphase roughness, i.e. a smooth irregularity of the interphase boundaries $\mathcal{S}_{ \pm}^{\varepsilon}$.

\subsection{Constitutive aspects: microcracks}

Many applications, in several engineering fields, request the modelling of quasi-brittle solid-solid interfaces. The brick/mortar interface in masonry structures can be considered a valid example, and additionally its modelling has a great scientific interest. A possible strategy to model brick/mortar interfaces is based on taking into account for a microcracking phenomenon in the constitutive equations of the material interphase, assumed to be a third material sandwiched between bricks and mortar joints (Rekik and Lebon, 2010, 2012). The interphase is assumed to be orthotropic and weakened by one family of parallel rectilinear microcracks with length 21 and orientation $\phi=\left(\mathbf{e}_{1}, \mathbf{t}\right)=0^{\circ}$ (see Figure 1). The adopted strategy is to recover the interphase effective elastic properties through an homogenisation approach. The formulation which follows is derived within the assumption of plane stress condition, with principal plane $\left(\mathbf{e}_{1}, \mathbf{e}_{3}\right)$. 
Figure 1 Sketch of the $\varepsilon$-thick representative elementary area (REA) taken into account in the homogenisation process, according the brick/mortar interface model by Rekik and Lebon $(2010,2012)$ (see online version for colours)

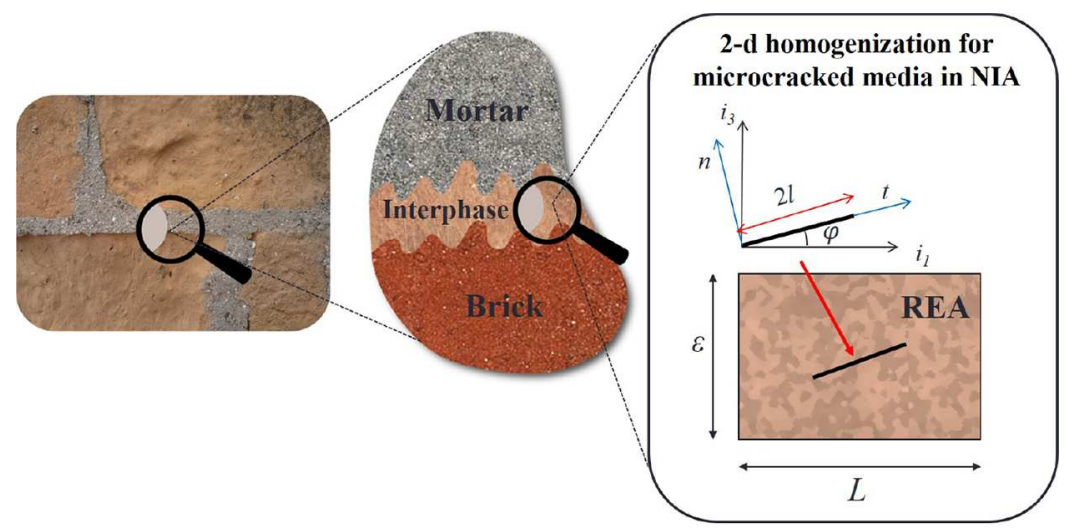

Former papers (Kachanov, 1994; Tsukrov and Kachanov, 2000; Kachanov and Sevostianov, 2005; Sevostianov and Kachanov, 2013) proposed a micromechanical homogenisation method for microcracked media under the non-interacting approximation (NIA). This homogenisation approach, defined also NIA in stress-based approach, is applied to obtain the elastic coefficient $A_{3333}^{\varepsilon}$, on which the soft interface law Eq. (50) depends. Particularly, reference is herein made to the brick/mortar interface model by Rekik and Lebon $(2010,2012)$ in a revisited version (refer to Fouchal et al. (2014) for further details).

The adopted homogenisation technique is a potential-based formulation in terms of the complementary elastic potential. Within the NIA framework, the problem of quantitative characterisation of microstructures is reduced to find the proper microstructural parameter of inhomogeneities entering the effective compliance tensor of the homogenised material (Kachanov and Sevostianov, 2005). Generally, the concentration parameters of microcracks in the context of the elastic properties are identified through the structure of a perturbative term in the elastic potential: $\Delta f$. In what follows, the adopted homogenisation method is briefly recalled, for further details one can refer to Kachanov (1994); Mauge and Kachanov (1994); Tsukrov and Kachanov (2000); Kachanov and Sevostianov (2005); Sevostianov and Kachanov (2013).

Let $\Gamma \subset \mathcal{S}$ be the crack middle surface with $\Gamma \perp \mathbf{e}_{3}$, and let $\mathbf{u}^{+}$and $\mathbf{u}^{-}$be the displacements at the parallel-to- $\mathcal{S}$ crack boundaries (recall that $\mathcal{S}$ has been taken to denote the interface among the adherents). Denote also as $\mathbf{u}_{\text {cod }}=\left\langle\mathbf{u}^{+}-\mathbf{u}^{-}\right\rangle=\left[\int_{\Gamma}\left(\mathbf{u}^{+}-\right.\right.$ $\left.\left.\mathbf{u}^{-}\right) \mathrm{d} \Gamma\right] /|\Gamma|$ the average measure of the displacement jump across the crack, in the following referred to as crack opening displacement (COD) vector. By considering a planestress assumption under a frictionless condition along crack faces, $\mathbf{u}_{\text {cod }}$ can be expressed in terms of the stress vector $\mathbf{T}_{3}=\boldsymbol{\sigma} \cdot \mathbf{e}_{3}$ as (Kachanov, 1994):

$$
\mathbf{u}_{\text {cod }}=\mathbf{B} \cdot \mathbf{T}_{3}
$$

where $\mathrm{B}$ is called COD tensor. By denoting with indexes $N$ and $T$ the normal and the tangential directions to the interface, respectively, the tensor $\mathbf{B}$ can be expressed as: $\mathbf{B}=$ $B_{N}\left(\mathbf{e}_{3} \otimes \mathbf{e}_{3}\right)+B_{T}\left(\mathbf{e}_{1} \otimes \mathbf{e}_{1}\right)$. It is worth remarking that this tensor has to be specialised with respect to the bulk material properties. Following the Eshelby approach (Eshelby, 
1961), the complementary elastic potential $f(\boldsymbol{\sigma})$ of the effective microcracked material results in:

$$
f(\boldsymbol{\sigma})=f_{0}(\boldsymbol{\sigma})+\Delta f=\frac{1}{2} \boldsymbol{\sigma}: \mathbb{S}_{0}: \boldsymbol{\sigma}+\frac{|\Gamma|}{2 A} \boldsymbol{\sigma}:\left(\mathbf{e}_{3} \otimes \mathbf{u}_{\mathrm{cod}}\right)
$$

where $f_{0}(\boldsymbol{\sigma})$ is the complementary potential expressed in terms of the compliance tensor $\mathbb{S}_{0}$ of the undamaged interphase, $\Delta f$ is a perturbative term depending on the microstructural features of microcracks, $A(\varepsilon)$ is the interphase area, and $|\Gamma| \simeq l^{2}$ is the characteristic microcrack area (or volume in 3D case). From Eq. (52) the scalar microcracks density is defined (Bristow, 1960):

$$
\rho=\frac{l^{2}}{A(\varepsilon)}=\frac{l^{2}}{\varepsilon L}
$$

where $l$ is the half-length of the flat crack and $L$ a characteristic length of the interphase. It is worth recalling that in $3 \mathrm{D}$ problems this scalar quantity it is assumed to be equal to $\rho=\frac{l^{3}}{V(\varepsilon)}$, where $V(\varepsilon)$ is the interphase volume.

Due to Eq. (52), specialised for an orthotropic undamaged interphase (Mauge and Kachanov, 1994), the effective compliance tensor $\mathbb{S}$ of the microcracked interphase can be componentwise derived as:

$$
(\mathbb{S})_{i j k l}=\left(\mathbb{S}_{0}\right)_{i j k l}+(\Delta \mathbb{S})_{i j k l}=\frac{\partial^{2} f}{\partial \sigma_{i j} \partial \sigma_{k l}}
$$

where $\Delta \mathbb{S}$ is the contribution compliance tensor associated to $\Delta f$ and accounting for the crack features. As a result, the elasticity tensor $\mathbb{A}^{\varepsilon}$ can be easily derived as: $\mathbb{A}^{\varepsilon}=\mathbb{S}^{-1}$. The tensor $\mathbb{A}^{\varepsilon}$ depends on the interphase thickness $\varepsilon$ through the area $A$ in the microcracks density expression (Eq. (53)). Finally, the component $A_{3333}^{\varepsilon}$ reads as:

$$
A_{3333}^{\varepsilon}=\frac{E_{1} E_{3}}{E_{1}+2 B_{N} \rho E_{1} E_{3}-E_{3} \nu_{13}^{2}}
$$

with

$$
B_{N}=\frac{\pi}{2 \sqrt{E_{3}}} \sqrt{\frac{2}{\sqrt{E_{1} E_{3}}}+\frac{1}{G_{13}}-\frac{2 \nu_{13}}{E_{1}}}
$$

where $E_{1}, E_{3}, G_{13}$ and $\nu_{13}$ are the elastic constants of the undamaged interphase. It is worth pointing out that these latter can be obtained in terms of the elastic properties of the two materials in contact, as the result of a homogenisation step performed on the undamaged $\varepsilon$-thick representative elementary volume (Rekik and Lebon, 2010, 2012; Fouchal et al., 2014).

By introducing Eq. (53) in Eq. (55), after some algebra, is easily to highlight that the elastic property $A_{3333}^{\varepsilon}$ derived from NIA homogenisation technique, respects the softinterphase assumption, in details, it can be rewritten as follows:

$$
A_{3333}^{\varepsilon}=\varepsilon \hat{A}_{3333} \simeq\left(\frac{L}{2 B_{N} l^{2}}\right) \varepsilon
$$

By substituting expression (55) into the interphase law (50), the damage parameter (i.e., microcracking) at the interface level, represented by the length $l$, can be taken into account. Moreover, note that it is possible to introduce an evolution law of the microcracks length $l$ with respect to the external force (pressure, shear stress, etc..) as it has been done in Rekik and Lebon (2010, 2012); Fouchal et al. (2014); Raffa, Lebon and Vairo (2015). 


\subsection{Geometrical imperfection: roughness}

In this section, a general methodology is proposed to take into account a smooth roughness of the interphase boundaries. Two given positive-valued roughness functions $\eta^{ \pm} \in \mathrm{C}^{0}\left(S, \mathbb{R}^{2}\right)$, describing the surfaces between the adherents and the interphase $\mathcal{S}_{ \pm}^{\varepsilon}$, are introduced (Fouchal et al., 2014). According to the smooth-roughness assumption, these two functions are independent on $\varepsilon$. Therefore, the domains $\mathcal{B}^{\varepsilon}, \Omega_{ \pm}^{\varepsilon}$ and the interfaces among adherents and adhesive $\mathcal{S}_{ \pm}^{\varepsilon}$ are re-defined as follows:

$$
\begin{aligned}
\mathcal{B}^{\varepsilon} & =\left\{\left(x_{1}, x_{2}, x_{3}\right) \in \Omega:-\frac{\varepsilon}{2} \eta^{-}\left(x_{1}, x_{2}\right)<x_{3}<\frac{\varepsilon}{2} \eta^{+}\left(x_{1}, x_{2}\right)\right\} \\
\Omega_{ \pm}^{\varepsilon} & =\left\{\left(x_{1}, x_{2}, x_{3}\right) \in \Omega: \pm x_{3}>\frac{\varepsilon}{2} \eta^{ \pm}\left(x_{1}, x_{2}\right)\right\} \\
\mathcal{S}_{ \pm}^{\varepsilon} & =\left\{\left(x_{1}, x_{2}, x_{3}\right) \in \Omega: x_{3}= \pm \frac{\varepsilon}{2} \eta^{ \pm}\left(x_{1}, x_{2}\right)\right\}
\end{aligned}
$$

As already done previously, let the following changes of variables be introduced in the domains $\mathcal{B}^{\varepsilon}$ and $\Omega_{ \pm}^{\varepsilon}$ (the rescaled quantities are, as usual, indicated with $\hat{\cdot}$ and $\bar{\cdot}$, respectively):

$$
\begin{gathered}
\hat{\mathbf{p}}^{\eta}:\left(x_{1}, x_{2}, x_{3}\right) \mapsto\left(z_{1}, z_{2}, z_{3}\right)=\left(x_{1}, x_{2}, \frac{x_{3}}{\eta^{+} \varepsilon}\right), \quad x_{3} \geq 0 \\
\hat{\mathbf{p}}^{\eta}:\left(x_{1}, x_{2}, x_{3}\right) \mapsto\left(z_{1}, z_{2}, z_{3}\right)=\left(x_{1}, x_{2}, \frac{x_{3}}{\eta^{-} \varepsilon}\right), \quad x_{3} \leq 0 \\
\overline{\mathbf{p}}^{\eta}:\left(x_{1}, x_{2}, x_{3}\right) \mapsto\left(z_{1}, z_{2}, z_{3}\right)=\left(x_{1}, x_{2}, x_{3}+\frac{1}{2}\left(1-\eta^{+} \varepsilon\right)\right), \\
x_{3} \geq \frac{\eta^{+} \varepsilon}{2} \\
\overline{\mathbf{p}}^{\eta}:\left(x_{1}, x_{2}, x_{3}\right) \mapsto\left(z_{1}, z_{2}, z_{3}\right)=\left(x_{1}, x_{2}, x_{3}-\frac{1}{2}\left(1-\eta^{-} \varepsilon\right)\right), \\
x_{3} \leq-\frac{\eta^{-} \varepsilon}{2}
\end{gathered}
$$

Accordingly, in the adhesive one has $d x_{3}=\eta^{ \pm} d z_{3}$.

The asymptotic expansion procedure, explained in the previous section, is not substantially modified. Accordingly, the relevant fields are expanded following Eqs. (5). Thereby, after the introduction of the interphase roughness, a perfect contact condition on the surfaces $\mathcal{S}_{ \pm}^{\varepsilon}$ is assumed. The formulation until Eq. (29) is quite unchanged and, starting from Eq. (29), let the obtained relationship be recalled:

$$
\frac{1}{2} \hat{A}_{3333}\left(\left|\hat{u}_{i, 3}^{2}\right|^{2} \hat{u}_{i, 3}^{2}\right)=\bar{P}_{i 3}^{0}=\text { in } \mathcal{B}
$$

By solving with respect to $\hat{\mathbf{u}}_{3}^{2}$ and by integrating with respect to $z_{3}$, in a domain which, herein, depends on the roughness functions $\eta^{ \pm}$, one has:

$$
\left[\hat{\mathbf{u}}^{2}\right]=\frac{\eta}{\left(\frac{1}{2} \hat{A}_{3333}\right)^{1 / 3}} \frac{1}{\left|\overline{\mathbf{P}}^{0} \mathbf{e}_{3}\right|^{2 / 3}} \overline{\mathbf{P}}^{0} \mathbf{e}_{3}
$$

where $\eta=\frac{\eta^{+}+\eta^{-}}{2}$ is the average roughness function. By applying a Taylor expansion to $\mathbf{u}^{\varepsilon}$ (Eqs. (3)) and using the matching relations (Eqs. (42)) with the interface conditions 
(Eqs. (43)-(45)), by using Eq. (66) instead of the first equation of Eqs. (45), the final soft interface law in the case of rough interphase is finally obtained:

$$
\mathbf{P}^{\varepsilon} \mathbf{e}_{3}=\frac{A_{3333}^{\varepsilon}}{2 \eta^{3} \varepsilon^{3}}\left|\llbracket \mathbf{u}^{\varepsilon} \rrbracket\right|^{2} \llbracket \mathbf{u}^{\varepsilon} \rrbracket+o\left(\varepsilon^{1 / 3}\right)
$$

\section{A simple numerical benchmark}

A numerical benchmark is proposed to show the applicability of the imperfect-nonlinearinterface model detailed above and to make some comparisons with the linear interface model by Fouchal et al. (2014). A quite simple three-dimensional geometry is treated, in particular an unit brick $(210 \mathrm{~mm} \times 100 \mathrm{~mm} \times 50 \mathrm{~mm})$ joined with a mortar joint $(210 \mathrm{~mm} \times 100 \mathrm{~mm} \times 10 \mathrm{~mm})$. The composite system is assumed to be fixed on a flat rigid plane. The geometry and the boundary conditions are outlined in Figure 2. This simple model has been chosen to focus, in a more accurate way, on the behavior of the brick/mortar interface in the linear and in nonlinear cases.

Figure 2 Sketch of the three-dimensional model (on the left). The surface loaded with the incremental displacement is represented in blue and the red surface is fixed. On the right side, a detail of the free tetrahedral mesh is represented (see online version for colours)
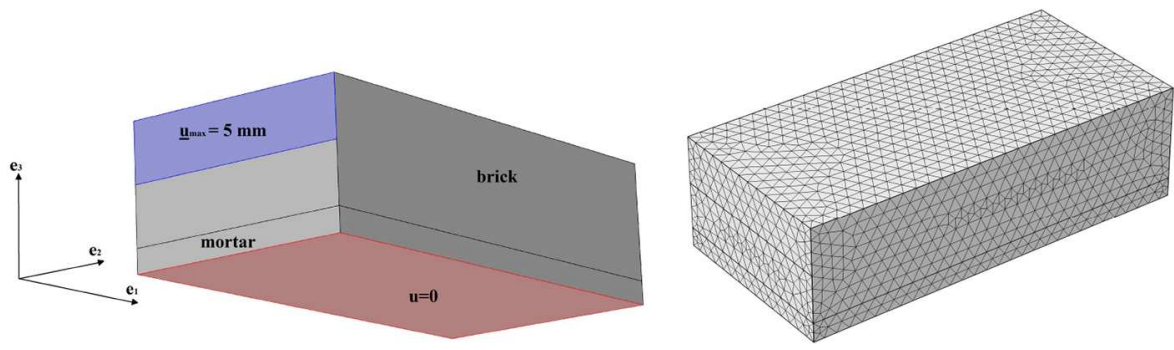

Within the hypothesis on the constitutive behavior of the principal constituents, i.e. brick and mortar, a linear and a nonlinear isotropic cases are considered. In the first case, the materials are assumed to be linearly elastic with parameters: Young modulus $E_{b}=$ $13 \times 10^{3} \mathrm{MPa}$ and Poisson ratio $\nu_{b}=0.25$ for the brick, and Young modulus $E_{m}=$ $4 \times 10^{3} \mathrm{MPa}$ and Poisson ratio $\nu_{m}=0.25$ for the mortar, respectively. Moreover, in the nonlinear case, both brick and mortar behave as hyperelastic materials of the St. Venant-Kirchhoff type with Lamé constants: $\lambda_{b}=3.6 \times 10^{3} \mathrm{MPa}$ and $\mu_{b}=5.4 \times 10^{3}$ $\mathrm{MPa}$ for the brick, and $\lambda_{m}=1.1 \times 10^{3} \mathrm{MPa}$ and $\mu_{m}=1.6 \times 10^{3} \mathrm{MPa}$ for the mortar, respectively.

The interphase is assumed to be a stratified layer comprising the material characteristics of both brick and mortar, i.e. it is a third material supposed to be transversely isotropic, whose elastic constants $E_{1}, E_{3}, G_{13}$ and $\nu_{13}$ are derived starting from the mechanical properties of the constituents $\left(E_{b}, E_{m}, \nu_{b}, \nu_{m}\right)$. To obtain the interphase elastic constants, a preliminary standard homogenisation for stratified is performed on the undamaged $\varepsilon$-thick representative elementary volume (for further details, see Appendix A in Rekik and Lebon (2012)). As a result, the following effective elastic constants are obtained: $E_{1}=8.5 \times 10^{3}$ $\mathrm{MPa}, E_{3}=6.4 \times 10^{3} \mathrm{MPa}, G_{13}=4.9 \times 10^{3} \mathrm{MPa}$ and $\nu_{13}=0.25$.

In addition, the implemented brick/mortar interface is assumed to derive from a microcracked interphase. To this aim, the microcracks density $\rho$ is derived according to 
Eq. (53), where the interphase area $A=\varepsilon \times L$ is calculated assuming a thickness value of $\varepsilon=0.2 \mathrm{~mm}$, a half-length value of the microcrack of $l=2 \mathrm{~mm}$, and $L=210 \mathrm{~mm}$ is the interphase length. As a result, a microcracks density of value $\rho=0.10$ is obtained.

Two typologies of imperfect interface are taken into account, the nonlinear interface proposed in this paper and a linear interface proposed by authors in former papers (Rekik and Lebon, 2010, 2012; Fouchal et al., 2014) to make some comparisons. The nonlinear imperfect interface is modelled according Eq. (50) in which the interphase stiffness $A_{3333}^{\varepsilon}$ is obtained by substituting in Eqs. (55) and (56) the values of the mechanical parameters of the undamaged interphase and the microcrack density $\rho$. As a consequence, the resulting stiffness is: $A_{3333}^{\varepsilon}=4.3 \times 10^{3} \mathrm{MPa}$.

For the linear-interface case, let consider the imperfect interface law correlating the Cauchy-stress vector in the normal-to-the-interface direction $\sigma \mathbf{e}_{3}$ and the displacementjump vector $\llbracket \mathbf{u} \rrbracket$ (see Klarbring (1991) and a generalisation for smooth roughness in Fouchal et al. (2014)):

$$
\boldsymbol{\sigma} \mathbf{e}_{3}=\frac{1}{\eta} \mathbf{K} \llbracket \mathbf{u} \rrbracket
$$

where $\eta$ is the average roughness function added to the standard asymptotic formulation by Fouchal et al. (2014), and the stiffness matrix K, whose components depend on the microcracks features and on the mechanical characteristics of the undamaged material, is expressed as follows (Fouchal et al., 2014):

$$
\mathbf{K}=\operatorname{diag}\left[K_{T} ; K_{N}\right]=\operatorname{diag}\left[\frac{L}{B_{T} l^{2}} ; \frac{L}{2 B_{N} l^{2}}\right]
$$

where the parameter $B_{N}$ given by Eq. (56), and parameter $B_{T}$ is given by a similar expression (Kachanov, 1994) which reads as:

$$
B_{T}=\frac{\pi}{2 \sqrt{E_{1}}} \sqrt{\frac{2}{\sqrt{E_{1} E_{3}}}+\frac{1}{G_{13}}-\frac{2 \nu_{13}}{E_{1}}}
$$

The obtained values of the stiffnesses in normal and in tangential-to-the-interface directions are: $K_{N}=5.9 \times 10^{4} \mathrm{~N} / \mathrm{mm}^{3}$ and $K_{T}=1.4 \times 10^{5} \mathrm{~N} / \mathrm{mm}^{3}$.

For the sake of simplicity, the roughness $\eta$ appearing in both linear and nonlinear interface laws (Eq. (68) and Eq. (67), respectively) is not taken into account in this numerical simulation and for further details on the effects of the roughness functions on the overall response of small masonries, one can refer to Fouchal et al. (2014).

Several numerical simulations are performed, aimed at validating the proposed interface model; in particular, in the model with the linearly elastic constituents, in what follows denoted as linear model, both the linear and the nonlinear interface laws, are implemented. In the nonlinear model, i.e. the one with the hyperelastic St. VenantKirchhoff type constituents, only the nonlinear interface law is considered. In addition, a linear and a nonlinear material behavior of the brick and the mortar are implemented with perfect interface condition (i.e. $\left.[[\mathbf{u}]]=0,\left[\left[\boldsymbol{\sigma} \mathbf{e}_{3}\right]\right]=0,\left[\left[\mathbf{P e}_{3}\right]\right]=0\right)$, in order to have some reference models.

All the analyses are performed with the COMSOL Multiphysics ${ }^{\circledR} 4.3$ software on a processor Intel(R) Core(TM) i3-2350M 2.3 GHz CPU. A free tetrahedral mesh of fine size is chosen in all the analyses cases, as represented in Figure 2. It is worth remarking that 
the brick/mortar interface is modelled through interface finite elements of zero thickness. The implemented numerical models aim to reproduce a push-out test on a single brick in a quasi-static loading process. The tests are performed in displacement-controlled mode with an imposed displacement of a maximum value equal to $5 \mathrm{~mm}$. The degrees of freedom and the solution times expressed in seconds are summarised in Table 1, for all the analysis cases.

Table 1 Values of degrees of freedom (dof) and solution times (in seconds) for all the analysed numerical model divided into sub-cases according the interface condition

\begin{tabular}{lccc}
\hline brick and mortar & interface & dof & CPU (s) \\
\hline \multirow{3}{*}{ linear } & perfect & 213621 & 309 \\
& linear & 219822 & 326 \\
\multirow{2}{*}{ nonlinear } & nonlinear & 219822 & 9716 \\
& perfect & 213621 & 8831 \\
& nonlinear & 219822 & 10317 \\
\hline
\end{tabular}

It is worth noting that it could be possible to reduce the degrees of freedom and, consequently, the solution times, by applying some symmetry considerations. Nevertheless, the remarkable aspect is the large difference in terms of solution time among linear and nonlinear calculations, independently if the nonlinearity is localised at the interface level (nonlinear interface condition) or in the constituents (nonlinear model). Moreover, both in the linear and in the nonlinear model, the introduction of the linear and nonlinear-imperfect-interface condition, respectively, does not produce a significant increment of the solution times with respect the perfect-interface cases.

The numerical simulations stop when the imposed displacement reaches its maximum value $(5 \mathrm{~mm})$. In Figure 3 a deformed shape at the final configuration is shown and the distribution of displacement field is mapped.

Figure 3 Final deformed shape relative to the linear model with the nonlinear interface condition (on the right). The $x_{1}$-component of the displacement field is mapped in colors. Final deformed shape of the interface in the same model (on the left) with color map of the $x_{3}$-component of the displacement-jump vector (a factor scale of 5 is applied) (see online version for colours)
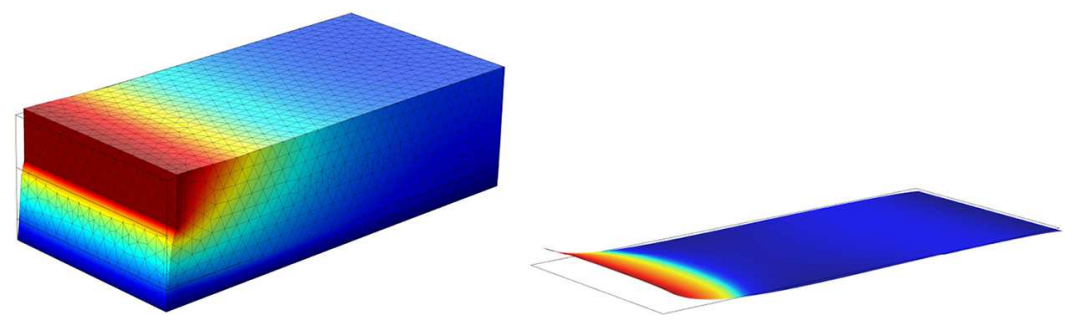

The curves shown in Figure 4 represent the $x_{1}$-component of the reaction force (i.e., in the direction of the imposed displacement) resulting from the loaded boundary, plotted with respect to the $x_{1}$-component of the displacement jump averaged over the interface surface, for all the analysed cases. Interestingly, both the linear model with the nonlinear interface 
and the nonlinear model with the nonlinear interface, allow to take into account for larger deformations (about one order of magnitude) at the interface level, than the linear model with the linear interface.

Figure 4 Reaction force in the $x_{1}$-direction averaged over the loaded boundary surface vs. the $x_{1}$-component of the average displacement-jump vector. Comparison among: the linear model with the linear interface (- $\triangle--)$; the linear model with the nonlinear interface $(-\star-)$; and the nonlinear model with the nonlinear interface (- - $\square--)$. A zoom of the curve relative to the linear model with linear interface is represented

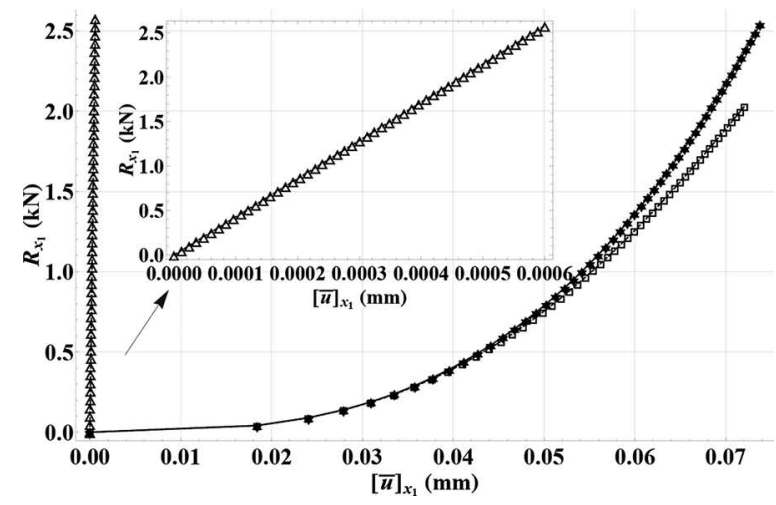

A comparison of Figures 5 and 6 put in evidence this aspect. The two figures represent the distribution, at the final configuration, of the $x_{1}$-component and of the $x_{3}$-component of the displacement-jump vector, respectively, along a cut line obtained from the intersection of the interface plane with the plane of symmetry.

Figure 5 Final distribution of the $x_{1}$-component of the average displacement-jump vector along the interface in the $x_{1}$-direction (recall that the maximum value of the imposed displacement is $5 \mathrm{~mm}$ ). Comparison among: the linear model with linear interface $(--\triangle--)$; the linear model with nonlinear interface $(-\star-)$; and the nonlinear model with nonlinear interface (-- $\square--$ ). A zoom of the curve relative to the linear model with the linear interface is represented

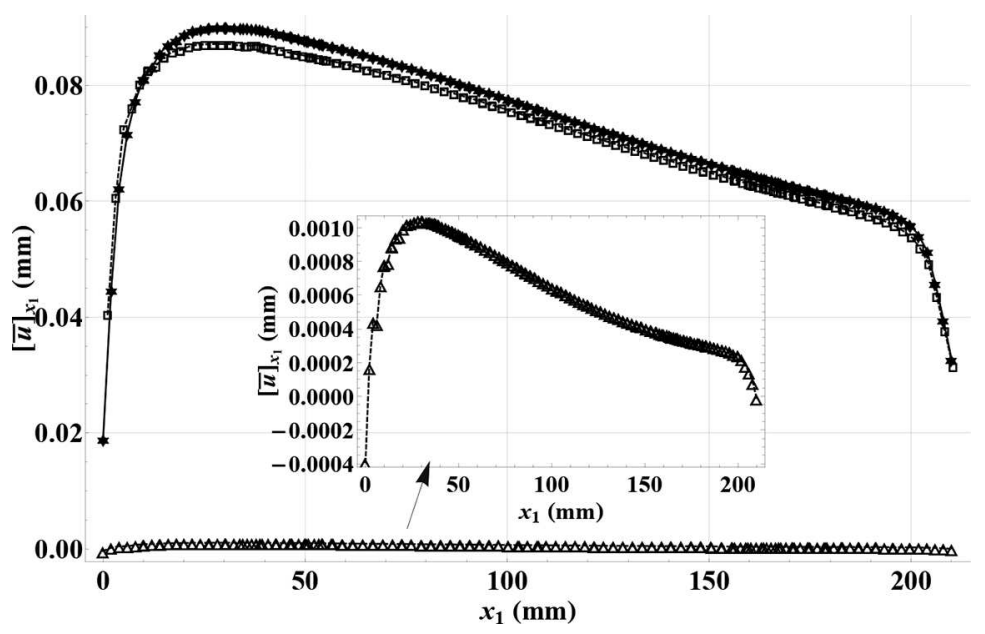


Figure 6 Final distribution of the $x_{3}$-component of the average displacement-jump vector along the interface in the $x_{1}$-direction (recall that the maximum value of the imposed displacement is $5 \mathrm{~mm}$ ). Comparison among: the linear model with the linear interface $(--\triangle--)$; the linear model with the nonlinear interface $(-\star-)$; and the nonlinear model with the nonlinear interface (- $\square--$ ). A zoom of the curve relative to the linear model with the linear interface is represented

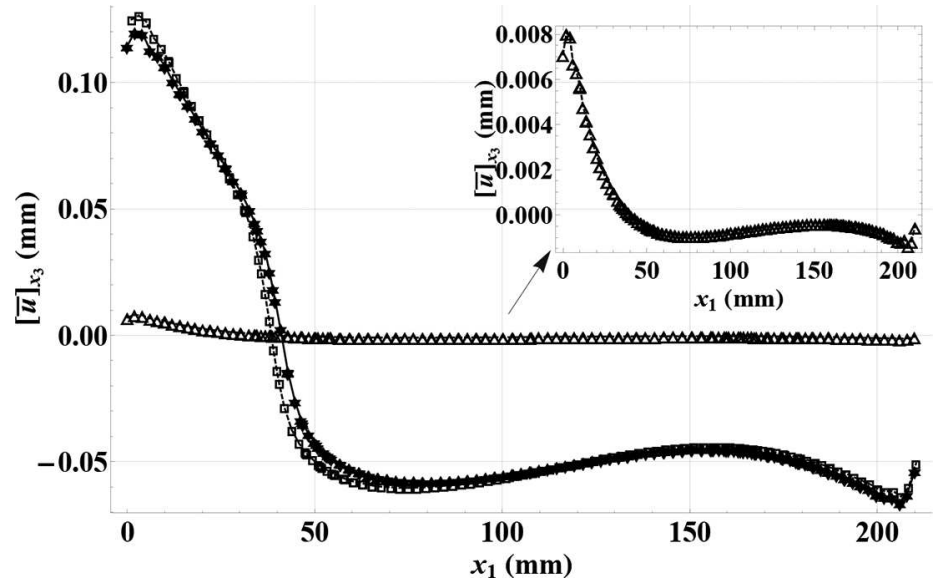

Furthermore, Figures 4-6 highlight that is not strictly necessary to model the adherents as hyperelastic materials in order to take into account the geometrical nonlinearities, i.e. large deformation, in terms of global response. In fact, the implementation of a nonlinear imperfect interface, as the proposed St. Venant Kirchhoff model, in a linearly elastic composite system, is sufficient to catch in an accurate way the nonlinear-interface behavior as the fully nonlinear model, reaching the same order of magnitude in terms of displacement jumps.

Figure 7 Von Mises stress (MPa) in the linear model with the nonlinear interface, with a particular of the stress distribution at the interface level (see online version for colours)

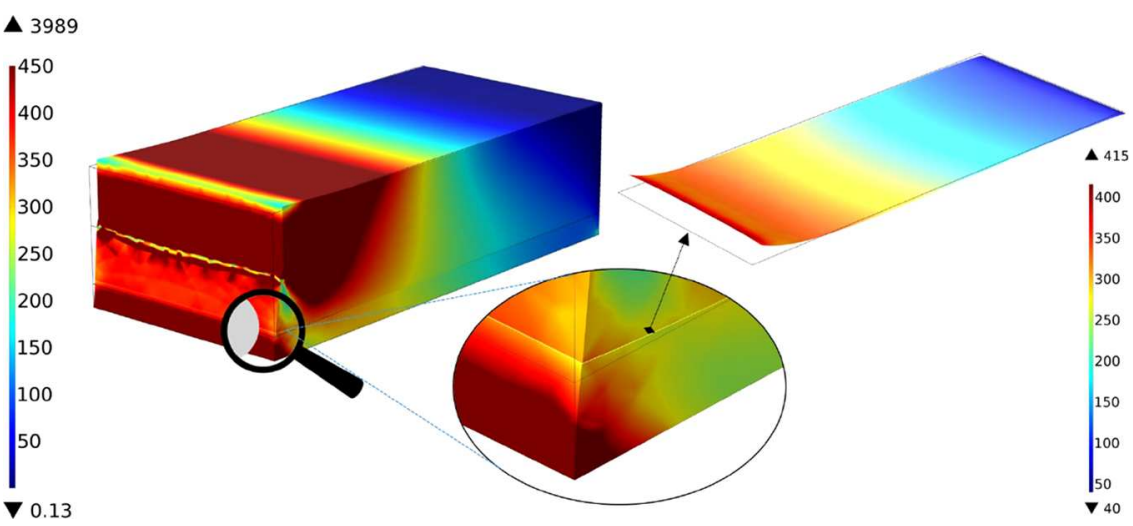

Figures 7-8 represent the distribution of the Von Mises stresses in the linear model with the nonlinear interface and in the nonlinear model with the nonlinear interface, in both cases a detail of the interface zone is represented. It is worth noting, from Figures 7-8, the difference in terms of magnitude of the stresses. In particular, in the nonlinear model with 
the nonlinear interface the Von Mises stresses are significantly smaller than in the linear model. Moreover, by analyzing the particular of the interfaces in both model, a significant difference in terms of stress distribution can be appreciated.

Figure 8 Von Mises stress (MPa) in the nonlinear model with the nonlinear interface, with a particular of the stress distribution at the interface level (see online version for colours)

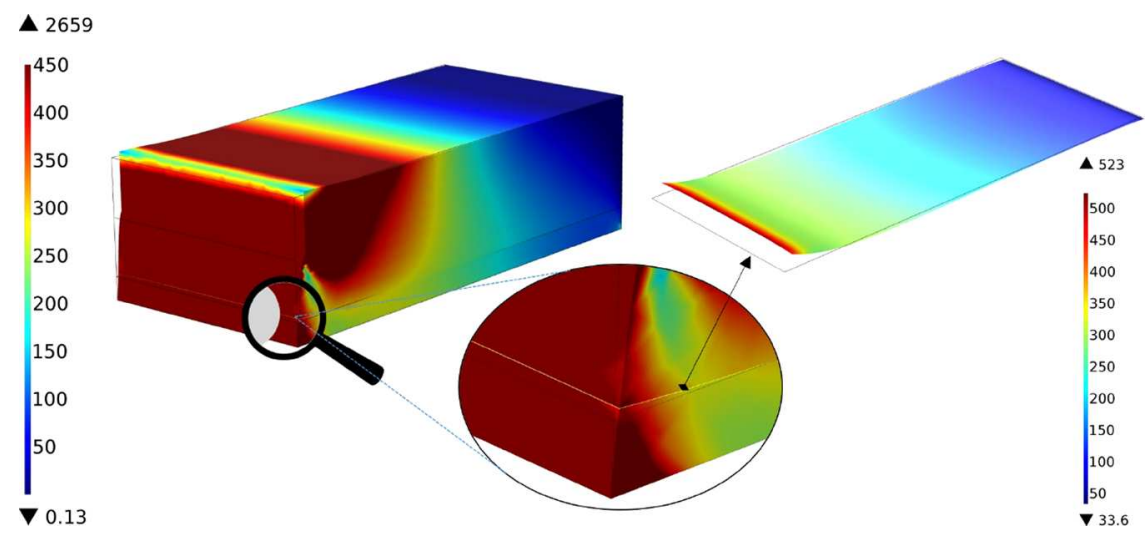

\section{Conclusions}

The proposed nonlinear-imperfect-interface model, formulated within the finite-strain theory, is derived from an interphase comprising a hyperelastic material of the St. VenantKirchhoff type. Two model enhancements have been introduced, aimed at taking into account both for damage and for smooth geometrical imperfections of the interface surface. The obtained soft interface law has been used to implement the imperfect interface in a simple numerical three-dimensional benchmark.

The proposed imperfect interface model has been formulated to catch the pre-peak behavior of quasi-brittle interfaces. Accordingly, an application in modelling brick/mortar interface is considered. For the numerical validation, three cases have been compared. The first two models, defined as linear, have been modelled with linearly elastic adherents (brick and mortar), and with two different interface conditions. In the first case, the interface has been modelled with a linear interface law available in literature (Rekik and Lebon, 2010, 2012; Fouchal et al., 2014), and in the second case, the proposed nonlinear interface law has been implemented. The third model, defined as nonlinear, is a fully nonlinear one, in which the adherents have been modelled with a St. Venant - Kirchhoff hyperelastic material and the interface has been modelled with the nonlinear-imperfectinterface law. Some comparisons have been carried out in terms of displacement jumps and of stresses distribution.

The soundness and the consistency of the proposed model are highlighted, both from a theoretical and a numerical points of view. Moreover, it has been established that the linear model with the nonlinear interface is able to catch the large displacements occurring at the interface level as much as the fully nonlinear model, additionally, the computational cost is smaller in the first case (see Table 1). It is worth remarking that the proposed interface law is able to describe a nonlinear pre-peak behavior of the imperfect interfaces (one refers 
to Figure 4). In this study, such a method can be able to describe the constitutive interface behavior of other types of quasi-brittle materials. Finally, the proposed model is believed to be an useful tool in studying large displacements effects in small masonry assemblies. Nevertheless, several numerical simulations are outgoing to investigate the influence of such an imperfect interface on masonry panels. Evidently, it will be taken into account that the introduction of a nonlinear interface laws, causes a significant increment of the computational costs with respect to the linear interface case. These numerical simulations will be the subject of the second part of this work.

\section{Acknowledgement}

MLR work was supported by Bando Vinci 2013 (n. C2-73) of Università Italo-Francese (UIF). RR thanks the financial support from ENDIF through the "Fondo per necessità di base della ricerca-2014".

\section{References}

Abdelmoula, R., Coutris, M. and Marigo, J.J. (1998) 'Comportement asymptotique d'une interface mince', C. R. Acad. Sci. IIB, Vol. 326, No. 4, pp.237-242.

Alfano, G. and Sacco, E. (2006) 'Combining interface damage and friction in a cohesive-zone model', Int. J. Num. Meth. Eng., Vol. 68, No. 5, pp.542-582.

Ascione, F. (2009a) 'Mechanical behaviour of FRP adhesive joints: a theoretical model', Compos. Part B-Eng., Vol. 40, pp.116-124.

Ascione, F. (2009b) 'Ultimate behaviour of adhesively bonded FRP lap joints'. Compos. Part B-Eng., Vol. 40, pp.107-115.

Ascione, F., Mancusi, G., Spadea, S., Lamberti, M., Lebon, F. and Maurel-Pantel, A. (2015) 'On the flexural behaviour of GFRP beams obtained by bonding simple panels: an experimental investigation', Compos. Struct., Vol. 131, pp.55-65.

Avila-Pozos, O., Klarbring, A. and Movchan, A.B. (1999) 'Asymptotic model of orthotropic highly inhomogeneous layered structure', Mech. Mat., Vol. 31, No. 2, pp.101-116.

Basilio, I., Fedele, R. , Louren,ço P.B. and Milani, G. (2014) 'Assessment of curved FRP-reinforced masonry prisms: Experiments and modeling', Constr. Build. Mater., Vol. 51, pp.492-505.

Benveniste, Y. (2006) 'An $O\left(h^{N}\right)$ interface model of a three-dimensional curved interphase in conduction phenomena', Proc. R. Soc. A, Vol. 462, pp.1593-1617.

Briseghella, L., Pavan, P. and Secchi, S. (1998) 'Damage and fracture in brittle materials', Comp. Meth. Struct. Masonry., Vol. 4, pp.59-66.

Bristow, J.R. (1960) 'Microcracks, and the static and dynamic elastic constants of annealed and heavily cold-worked metals', Brit. J. Appl. Phys., Vol. 11, No. 2, pp.81-85.

Calderini, C. and Lagomarsino, S. (2008) 'Continuum model for in-plane anisotropic inelastic behavior of masonry', J. Struct. Eng.-Asce, Vol. 134, No. 2, pp.209-220.

Cecchi, A. and Sab, K. (2002) 'A multi-parameter homogenization study for modeling elastic masonry', Eur. J. Mech. A/Solids, Vol. 21, No. 2, pp.249-268.

Ceroni F., de Felice G., Grande, E., Malena M., Mazzotti, C., Murgo, F., Sacco, E. and Valluzzi, M.R. (2014) 'Analytical and numerical modeling of composite-to-brick bond', Mater. Struct., Vol. 47, No. 12, pp.1987-2003.

Ciarlet, P.G. (1997) 'Mathematical elasticity', in Ciarlet, P.G. (Ed.): Mathematical Elasticity Volume II: Theory of Plates. Vol. 27 of Studies in Mathematics and Its Applications, Elsevier, pp.VII - XI, North Holland. 
de Buhan, P. and de Felice, G. (1997) 'A homogenization approach to the ultimate strength of brick masonry', J. Mech. Phys. Sol., Vol. 45, No. 7, pp.1085-1104

Drosopoulosa, G.A., Stavroulakis, G.E. and Massalasa, C.V. (2006) 'Limit analysis of a single span masonry bridge with unilateral frictional contact interfaces', Eng. Struct., Vol. 28, No. 13, pp.1864-1873.

Dumont, S., Lebon, F. and Rizzoni, R. (2014) 'An asymptotic approach to the adhesion of thin stiff films', Mech. Res. Commun., Vol. 58, pp.24-35.

Eshelby, J.D. (1961) 'Elastic inclusions and inhomogeneities', Progress in Solid Mechanics, Vol. 2, North-Holland, Amsterdam, pp.87-140.

Fremond, M. (1987) ‘Adhesion of solids', J. Mech Th. Appl., Vol. 6, No. 3, pp.383-407.

Fouchal, F., Lebon, F., Raffa, M.L. and Vairo, G. (2014) 'An interface model including cracks and roughness applied to masonry', Open Civ. Eng. J., Vol. 8, pp.263-271.

Fouchal, F., Lebon, F. and Titeux, I. (2009) 'Contribution to the modelling of interfaces in masonry construction', Const. Build. Mat., Vol. 23, No. 6, pp.2428-2441.

Gabor, A., Bennani, A., Jacquelin, E. and Lebon, F. (2006). 'Modelling approaches of the in-plane shear behaviour of unreinforced and FRP strengthened masonry panels', Comp. Struct., Vol. 74, pp.277-288.

Gambarotta, L. and Lagomarsino, S. (1997) 'Damage models for the seismic response of brick masonry shear walls .1. the mortar joint model and its applications', Earthq. Eng. Struct. Dyn., Vol. 26, No. 4, pp.423-439

Geymonat, G., Krasucki, F. and Lenci, S. (1999) 'Mathematical analysis of a bonded joint with a soft thin adhesive', Math. Mech. Solid, Vol. 4, No. 2, pp.201-225.

Giambanco, G. and Di Gati, L. (1997) 'A cohesive interface model for the structural mechanics of block masonry’, Mech. Res. Commun., Vol. 24, No. 5, pp.503-512.

Kachanov, M. (1994) 'Elastic solids with many cracks and related problems', Adv. Appl. Mech., Vol. 30, pp.259-445.

Kachanov, M. and Sevostianov, I. (2005) 'On quantitative characterization of microstructures and effective properties', Int. J. Solids Struct., Vol. 42, No. 2, pp.309-336.

Klarbring, A. (1991) 'Derivation of a model of adhesively bonded joints by the asymptotic expansion method', Int. J. Eng. Sci., Vol. 29, No. 4, pp.493-512.

Krasucki, F., Münch, A. and Ousset, Y. (2001) 'Analyse asymptotique d'un assemblage collé en élasticité non linéaire' C. R. Acad. Sci., Vol. 329, No. Série II b, pp.429-434.

Mauge, C. and Kachanov, M. (1994) 'Effective elastic properties of an anisotropic material with arbitrarily oriented interacting cracks', J. Mech. Phys. Solids, Vol. 42, pp.561-584.

Lebon, F. and Rizzoni, R. (2008) 'Asymptotic study of a soft thin layer: the non convex case', Mech. Adv. Mat. Struct., Vol. 15, No. 1, pp.12-20.

Lebon, F. and Rizzoni, R. (2010) 'Asymptotic analysis of a thin interface: the case involving similar rigidity’, Int. J. Eng. Sci., Vol. 48, No. 5, pp.473-486.

Lebon, F. and Rizzoni, R. (2011) 'Asymptotic behavior of a hard thin linear interphase: an energy approach', Int. J. Solids Struct., Vol. 48, pp.441-449.

Lebon, F., Rizzoni, R. and Ronel-Idrissi, S. (2004) 'Analysis of non-linear soft thin interfaces', Comput. Struct., Vol. 82, pp.1929-1938.

Lebon, F. and Zaittouni, F. (2010) 'Asymptotic modelling of interface taking into account contact conditions: Asymptotic expansions and numerical implementation', Int. J. Eng. Sci., Vol. 48, No. 2, pp.111-127.

Lotfi, H. and Shing, P. (1994) 'Interface model applied to fracture of masonry structures', J. Struct. Eng.-Asce, Vol. 120, No. 1, pp.63-80.

Lourenço, P. and Rots, J. (1997) 'Multisurface interface model for analysis of masonry structures', J. Eng. Mech., Vol. 123, No. 7, pp.660-668. 
Lu Y., Huang J.Y., Wang C., Sun S. and Lou J. (2010) 'Cold welding of ultrathin gold nanowires', Nat. Nanotechnol., Vol. 5, pp.213-224.

Milani, G., Lourenço, P.B. and Tralli, A. (2006) 'Homogenised limit analysis of masonry walls, Part I: failure surfaces', Comp. Struct., Vol. 84, Nos. 3-4, pp.166-180.

Monerie, Y. and Raous, M. (2000) 'A model coupling adhesion to friction for the interaction between a crack and a fibre/matrix interface', Zeitschr. Angew. Math. Mech., Vol. 80, pp.S205-S208.

Page, A. (1978) 'Finite element model for masonry', J. Struct. Div., Vol. 104, pp.1267-1285.

Pelissou, C. and Lebon, F. (2009) 'Asymptotic modeling of quasi-brittle interfaces', Comput. Struct., Vol. 87, Nos. 19-20, pp.1216-1223.

Raffa, M.L., Lebon, F. and Vairo, G. (2015) 'Normal and tangential stiffnesses of rough surfaces in contact via an imperfect interface model', Int. J. Solid. Struct., in press.

Raous, M., Cangemi, L. and Cocu, M. (1999) 'A consistent model coupling adhesion, friction, and unilateral contact', Comput. Meth. Appl. Mech. Eng., Vol. 177, Nos. 3-4, pp.383-399.

Rekik A. and Lebon, F. (2010) 'Identification of the representative crack length evolution in a multilevel interface model for quasi-brittle masonry', Int. J. Solids Struct., Vol. 47, Nos. 22-23, pp.3011-3021.

Rekik A. and Lebon, F. (2012) 'Homogenization methods for interface modeling in damaged masonry', Adv. Eng. Software, Vol. 46, No. 1, pp.35-42.

Rizzoni, R., Dumont, S. and Lebon, F. (2015) 'On Saint Venant - Kirchhoff Imperfect Interfaces', submitted.

Rizzoni, R., Dumont, S., Lebon, F. and Sacco, E. (2014) 'Higher order model for soft and hard elastic interfaces', Int. J. Solids Struct., Vol. 51, Nos. 23-24, pp.4137-4148.

Rizzoni, R. and Lebon, F. (2013) 'Imperfect interfaces as asymptotic models of thin curved elastic adhesive interphases', Mech. Res. Commun., Vol. 51, pp.39-50.

Scimemi, G., Giambanco, G. and Spada, A. (2014) 'The interphase model applied to the analysis of masonry structures', Comp. Meth. Appl. Mech. Eng., Vol. 279, pp.66-85.

Sevostianov, I. and Kachanov, M. (2013) 'Non-interaction approximation in the problem of effective properties', in Kachanov, M., Sevostianov, I. (Eds.): Effective Properties of Heterogeneous Materials. Vol. 193 of Solid Mechanics and Its Applications, Springer, The Netherlands, pp.1-95.

Spada, A., Giambanco, G. and Rizzo, P. (2009) 'Damage and plasticity at the interfaces in composite materials and structures', Comput. Meth. Appl. Mech. Eng., Vol. 198, No. 49-52, pp.3884-3901.

Tsukrov, I. and Kachanov, M. (2000) 'Effective moduli of an anisotropic material with elliptical holes of arbitrary orientational distribution', Int. J. Solid. Struct., Vol. 37, No. 41, pp.5919-5941.

Zhang, W. and Bay, N. (1997) 'Cold welding - theoretical modeling of the weld formation', Weld. J., Vol. 76 , No. 10, pp.417s-420s. 